\title{
HIGH-PRECISION RADIO AND INFRARED ASTROMETRY OF LSPM J1314+1320AB. II. TESTING PRE- MAIN-SEQUENCE MODELS AT THE LITHIUM DEPLETION BOUNDARY WITH DYNAMICAL MASSES*
}

\author{
Trent J. Dupuy ${ }^{1}$, Jan Forbrich ${ }^{2,3}$, Aaron Rizzuto ${ }^{1}$, Andrew W. ManN ${ }^{1}$, Kimberly Aller ${ }^{4}$, Michael C. Liu ${ }^{4}$, \\ AdAM L. Kraus ${ }^{1}$, AND Edo Berger ${ }^{3}$ \\ ${ }^{1}$ The University of Texas at Austin, Department of Astronomy, 2515 Speedway C1400, Austin, TX 78712, USA \\ ${ }^{2}$ University of Vienna, Department of Astrophysics, Türkenschanzstr. 17, A-1180 Vienna, Austria \\ ${ }^{3}$ Harvard-Smithsonian Center for Astrophysics, 60 Garden Street, Cambridge, MA 02138, USA \\ ${ }^{4}$ Institute for Astronomy, University of Hawai ${ }^{i}$, 2680 Woodlawn Drive, Honolulu, HI 96822, USA \\ Received 2016 April 1; revised 2016 April 30; accepted 2016 May 20; published 2016 August 3
}

\begin{abstract}
We present novel tests of pre-main-sequence models based on individual dynamical masses for the M7 binary LSPM J1314+1320AB. Joint analysis of Keck adaptive optics astrometric monitoring along with Very Long Baseline Array radio data from a companion paper yield component masses of $92.8 \pm 0.6 M_{\text {Jup }}$ $\left(0.0885 \pm 0.0006 M_{\odot}\right)$ and $91.7 \pm 1.0 M_{\text {Jup }}\left(0.0875 \pm 0.0010 M_{\odot}\right)$ and a parallactic distance of $17.249 \pm 0.013 \mathrm{pc}$. We find component luminosities consistent with the system being coeval at $80.8 \pm 2.5 \mathrm{Myr}$, according to BHAC15 evolutionary models. The presence of lithium is consistent with model predictions, marking the first test of the theoretical lithium depletion boundary using ultracool dwarfs of known mass. However, we find that the evolutionary model-derived average effective temperature $(2950 \pm 5 \mathrm{~K})$ is $180 \mathrm{~K}$ hotter than that given by a spectral type- $T_{\text {eff }}$ relation based on BT-Settl models $(2770 \pm 100 \mathrm{~K})$. We suggest that the dominant source of this discrepancy is model radii being too small by $\approx 13 \%$. In a test mimicking the typical application of models by observers, we derive masses on the H-R diagram using luminosity and BT-Settl temperature. The estimated masses are lower by $46_{-19}^{+16} \%(2.0 \sigma)$ than we measure dynamically and would imply that this is a system of $\approx 50 M_{\text {Jup }}$ brown dwarfs, highlighting the large systematic errors possible in H-R diagram properties. This is the first time masses have been measured for ultracool $(\geqslant M 6)$ dwarfs displaying spectral signatures of low gravity. Based on features in the infrared, LSPM J1314+1320AB appears to have higher gravity than typical Pleiades and $\mathrm{AB}$ Dor members, opposite the expectation given its younger age. The components of LSPM J1314+1320AB are now the nearest, lowest mass pre-main-sequence stars with direct mass measurements.
\end{abstract}

Key words: astrometry - binaries: visual - parallaxes - stars: fundamental parameters - stars: individual (LSPM $\mathrm{J} 1314+1320)$ - stars: pre-main sequence

\section{INTRODUCTION}

A major goal of stellar astrophysics is to understand the early evolution of stars, before they reach a stable equilibrium on the main sequence. In theory, the fundamental parameters of mass, composition, and angular momentum uniquely determine the course of all stellar evolution, including the pre-main-sequence phase. Binary stars are perhaps the most useful empirical calibrators available for testing stellar models because their components share a common age and composition, and dynamical masses can be derived from their orbital motion. While dozens of mass measurements have been obtained for pre-main-sequence stars (e.g., see reviews from Hillenbrand \& White 2004 and Mathieu et al. 2007, p. 411; Gennaro et al. 2012; Stassun et al. 2014), most of these are for stars more massive than $0.5 M_{\odot}$. Steady progress has been made to push measurements to lower masses (e.g., Simon et al. 2000; Stassun et al. 2006; Kraus et al. 2015; Lodieu et al. 2015; David et al. 2016). However, there are still only a handful of masses measured for stars at or below the $0.2-0.3 M_{\odot}$ peak in the initial mass function (Bastian et al. 2010), leaving premain-sequence models for a large fraction of stars poorly constrained.

Data presented herein were obtained at the W. M. Keck Observatory, which is operated as a scientific partnership among the California Institute of Technology, the University of California, and the National Aeronautics and Space Administration. The Observatory was made possible by the generous financial support of the W. M. Keck Foundation.
Previous work to measure the masses of pre-main-sequence stars has mostly focused on star-forming regions, like the nearby Taurus-Auriga and Scorpius-Centaurus-Lupus-Crux complexes with ages of $\sim 1-10 \mathrm{Myr}$ and distances of $\sim 150 \mathrm{pc}$ (e.g., Schaefer et al. 2008; Czekala et al. 2016; Rizzuto et al. 2016). Over the last two decades, a growing number of young stars much closer to the Sun and with wider ranging ages ( 8-150 Myr) have been identified (e.g., Zuckerman \& Song 2004; Torres et al. 2008). The proximity of these stars offers many benefits, including the possibility of spatially resolving binaries with smaller semimajor axes and correspondingly shorter orbital periods for dynamical mass determinations.

LSPM J1314+1320 was first identified as a star exhibiting

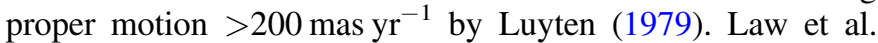
(2006) included it as a candidate late-type star in their high angular resolution survey using lucky imaging at $i^{\prime}$ and $z^{\prime}$ bands, where their sample was selected from objects with red $V-K$ colors in the LSPM catalog (Lépine \& Shara 2005). Law et al. (2006) estimated a spectral type of M6 for LSPM J1314 +1320 from its $V-K$ color and discovered that it was a binary with a separation of 130 mas and flux ratios of $\Delta i^{\prime}=0.93 \pm 0.25 \mathrm{mag}$ and $\Delta z^{\prime}=0.97 \pm 0.25 \mathrm{mag}$. Meanwhile, Lépine (2005) had identified LSPM J1314+1320 as a potential nearby star with an estimated distance of $9.7 \mathrm{pc}$, leading them to obtain spectra and astrometry that revealed a spectral type of $\mathrm{M} 7, \mathrm{H} \alpha$ in emission, and a parallactic distance 

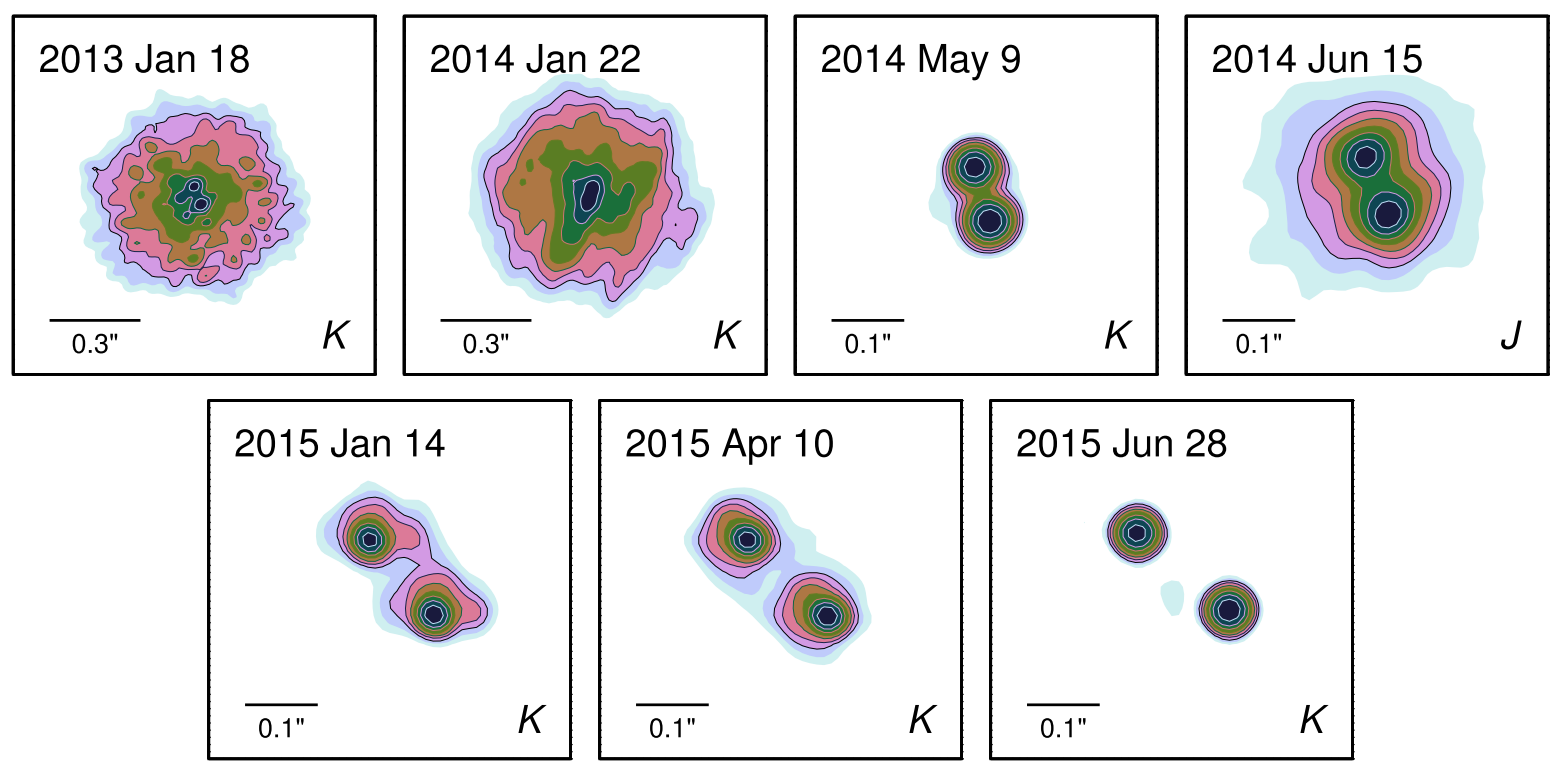

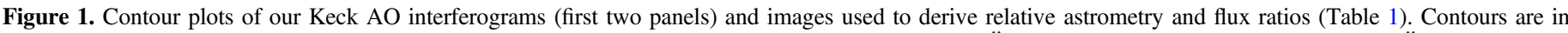

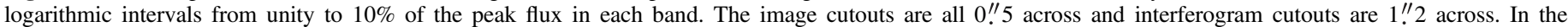

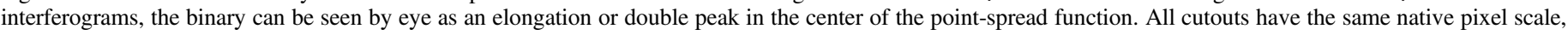
and here we have rotated them so that north is up.

of $16.4 \pm 0.8 \mathrm{pc}$ (Lépine et al. 2009). They concluded that their original photometric distance estimate was much smaller than the parallactic distance due to unresolved binarity and/or extreme youth.

The first definitive evidence for the youth of LSPM J1314 $+1320 \mathrm{AB}$ came from the analysis by Schlieder et al. (2014) of its published optical spectra and Schlieder et al.'s own nearinfrared (NIR) spectrum, all spatially unresolved. These spectra display weak alkali lines, which are indicative of low surface gravity, and strong Li I absorption. Although Schlieder et al. (2012) had previously identified LSPM J1314+1320AB as a likely new member of the $\mathrm{AB}$ Doradus moving group $(\sim 150 \mathrm{Myr})$, subsequent analysis and updated proper motion data has led to the conclusion that it cannot be confidently associated with any known group (Gagné et al. 2014; Schlieder et al. 2014). McLean et al. (2011) has also identified LSPM J1314+1320AB as a source of bright ( 1 mJy), persistent radio emission with a flat spectrum across a wide range of frequencies $(1.43-22.5 \mathrm{GHz})$, suggesting high levels of magnetic activity and a stable, large-scale magnetosphere (Williams et al. 2014, 2015). Therefore, LSPM J1314 $+1320 \mathrm{AB}$ is potentially useful as a benchmark not only for evolutionary models, but also for models of stellar magnetism.

We present here spatially resolved relative astrometric monitoring of LSPM J1314+1320AB obtained with Keck $\mathrm{AO}$ that allows us to determine its orbit and thereby its dynamical total mass $\left(M_{\mathrm{tot}}\right)$. We combine our Keck data with spatially resolved Very Long Baseline Array (VLBA) absolute astrometry from a companion paper (Forbrich et al. 2016; hereinafter Paper I) to simultaneously model the orbital motion, proper motion, and parallax and thereby derive individual dynamical masses of $\lesssim 1 \%$ precision. We confirm that LSPM J1314+1320AB is unambiguously in the pre-mainsequence phase of its evolution and use our dynamical masses to perform tests of models, examining properties such as luminosity, temperature, and lithium depletion.

\section{OBSERVATIONS}

\subsection{Keck/NIRC2 Astrometry}

We have been monitoring the resolved orbital motion of both components of LSPM J1314+1320AB using Keck adaptive optics (AO) with the facility NIR camera NIRC 2 . We used both direct imaging and non-redundant aperture masking to measure the binary's separation, position angle (PA), and flux ratio in $J$ and $K$ bands. Typical examples of our images and masking interferograms from each epoch are shown in Figure 1. An analysis of the masking data was done using a pipeline similar to that in previous papers containing NIRC2 masking data (e.g., Ireland et al. 2008; Ireland \& Kraus 2008) and is described in detail in Section 2.2 of Dupuy et al. (2009b). For imaging data, we used the same methods described in our previous work (e.g., see Dupuy et al. 2009a, 2016). Briefly, after a standard reduction of the images (dark subtraction and flat fielding) we fit a three-component two-dimensional Gaussian model to the two binary components. For the last three epochs, when the binary separation was widest, we were able to use the StarFinder package (Diolaiti et al. 2000) instead. StarFinder iteratively solves for both the binary parameters and an image of the point-spread function. We then corrected our derived $(x, y)$ positions using the NIRC2 distortion solution of Yelda et al. (2010), which has a pixel scale of $9.952 \pm 0.002$ mas pixel $^{-1}$ and a $+0.252 \pm 0^{\circ} 0.009$ offset added to the orientation given in the NIRC2 image headers. The last epoch was obtained after a major realignment of the Keck AO system on 2015 April 13. Therefore, for this epoch, we used an updated distortion solution from Service et al. (2016), which has a pixel scale of $9.971 \pm 0.004$ mas pixel $^{-1}$ and an orientation of $+0^{\circ} .262 \pm 0^{\circ} .0 .020$.

Table 1 summarizes the resolved astrometry and flux ratios derived from our Keck observations. As an estimate of our uncertainties, we used the rms of the best-fit binary parameters at a given epoch across the multiple images or masking interferograms. To vet these errors, we performed a standard 
Table 1

Keck/NIRC2 Adaptive Optics Astrometry for LSPM J1314+1320AB

\begin{tabular}{|c|c|c|c|c|c|}
\hline Date (UT) & Filter & Separation (mas) & $\mathrm{PA}\left({ }^{\circ}\right)$ & $\Delta m$ (mag) & Note \\
\hline 2013 Jan 18 & $K$ & $66.2 \pm 0.5$ & $202.3 \pm 0.5$ & $0.092 \pm 0.012$ & masking \\
\hline 2014 Jan 22 & $K$ & $54.0 \pm 1.9$ & $342 \pm 7$ & $0.048 \pm 0.024$ & masking \\
\hline 2014 May 9 & $K$ & $78.62 \pm 0.21$ & $15.2 \pm 0.6$ & $0.11 \pm 0.04$ & imaging \\
\hline 2014 Jun 15 & $J$ & $88.1 \pm 0.5$ & $21.7 \pm 0.5$ & $0.08 \pm 0.04$ & imaging \\
\hline 2015 Jan 14 & $K$ & $137.25 \pm 0.19$ & $41.55 \pm 0.04$ & $0.091 \pm 0.006$ & imaging \\
\hline 2015 Apr 10 & $K$ & $153.16 \pm 0.08$ & $46.27 \pm 0.03$ & $0.068 \pm 0.005$ & imaging \\
\hline 2015 Jun 28 & $K$ & $166.43 \pm 0.13$ & $49.84 \pm 0.05$ & $0.081 \pm 0.006$ & imaging \\
\hline
\end{tabular}

seven-parameter orbit least-squares fit (see Section 3.1 of Dupuy et al. 2010) to our Keck astrometry. We found a value of $\chi^{2}=3.5$ for 7 degrees of freedom (dof), which has a probability of $p\left(\chi^{2}\right)=0.83$, so we concluded that our Keck astrometric errors are reasonable. ${ }^{5}$

Our $K$-band flux ratios are somewhat inconsistent with being constant across all epochs, with $\chi^{2}=12.4$ ( 6 dof). To achieve $p\left(\chi^{2}\right)=0.5$, a systematic error of $0.011 \mathrm{mag}$ added in quadrature is required. Therefore, in the following analysis, we use $\Delta K=0.080 \pm 0.022 \mathrm{mag}$, which is the weighted average after adding this error floor in quadrature to the individual values. We conservatively use the rms of the measurements as the uncertainty since it encompasses both the fitting errors and the potential for variability. Finally, we note that our infrared flux ratios seem to be at odds with the optical flux ratios of $\Delta i^{\prime}=0.93 \pm 0.25 \mathrm{mag}$ and $\Delta z^{\prime}=0.97 \pm$ 0.25 mag from Law et al. (2006). However, for another binary in their survey, LHS 1901AB, they also report very unequal flux ratios $\left(\Delta i^{\prime}=1.3 \pm 0.7 \mathrm{mag}\right.$ and $\Delta z^{\prime}=1.3 \pm 0.7 \mathrm{mag}$; Law et al. 2008) while AO imaging gives flux ratios in $J H K$ of $\approx 0.1 \mathrm{mag}$ (Montagnier et al. 2006; Dupuy et al. 2010). This suggests that the apparent discrepancy may simply be due to systematic errors in the flux ratios derived from Lucky imaging, so we do not use them in our analysis.

\subsection{UH $2.2 \mathrm{~m} /$ SuperNova Integral Field Spectrograph (SNIFS) and Infrared Telescope Facility (IRTF)/SpeX Spectroscopy}

We obtained optical and NIR spectra of LSPM J1314 $+1320 \mathrm{AB}$ as part of a larger follow-up program of nearby bright M dwarfs from Lépine \& Gaidos (2011). In the optical, we used the SNIFS (Aldering et al. 2002; Lantz et al. 2004) on the University of Hawaii $2.2 \mathrm{~m}$ telescope on Maunakea, Hawaii, on 2015 January 9 UT. SNIFS provides simultaneous coverage from 3200-9700 $\AA$ at a resolution of $R \simeq 1000$. Details of our SNIFS observations and reduction can be found in Bacon et al. (2001) and Gaidos et al. (2014), which we briefly summarize here. The pipeline detailed in Bacon et al. (2001) performs dark, bias, and flat-field corrections, cleans the data of bad pixels and cosmic rays, then fits and extracts the integral field unit spaxels into a one-dimensional spectrum. The Gaidos et al. (2014) reduction takes this spectrum and performs flux calibration and telluric correction based on white dwarf standards taken throughout the night and a model of the atmosphere above Maunakea (Buton et al. 2013). The final reduced spectrum of LSPM J1314+1320AB has a signal-tonoise ratio $(\mathrm{S} / \mathrm{N})$ of $>100 \mathrm{pixel}^{-1}$ redward of $6000 \AA$.

\footnotetext{
5 When we quote $p\left(\chi^{2}\right)$, it is the probability of obtaining a value for $\chi^{2}$ as high or higher than the observed value given the degrees of freedom.
}

We obtained an NIR spectrum of LSPM J1314+1320AB using the SpeX spectrograph (Rayner et al. 2003) at the NASA IRTF on Maunakea, Hawaii, on 2013 May 16 UT. Our observations were taken in the short cross-dispersed (SXD) mode using the 0 ". $3 \times 15^{\prime \prime}$ slit $(R \simeq 2000)$, yielding simultaneous coverage from $0.8-2.4 \mu \mathrm{m}$, with a small gap near $1.8 \mu \mathrm{m}$ due to non-overlapping SXD orders. The target was placed at two positions along the slit (A and B) and was observed in an ABBA pattern in order to subsequently subtract the sky background. Six exposures were taken this way, yielding an S/ $\mathrm{N}>200$ per pixel in the $H$ and $K$ bands. To correct for telluric lines, we observed an A0V star immediately after the target.

Our SpeX spectrum was extracted using the SpeXTool package (Cushing et al. 2004), which performs flat-field correction, wavelength calibration, sky subtraction, and extraction of the one-dimensional spectrum. Multiple exposures were combined using the $x$ combspec routine. A telluric correction spectrum was constructed from the A0V star and applied to the target spectrum using the xtellcor package (Vacca et al. 2003). Separate orders were stacked using the xcombspec tool, which also shifts the flux level in different orders to match each other. These corrections were $1 \%$ or less per order.

\section{RESULTS}

\subsection{MCMC Astrometric Orbit and Parallax Analysis}

We combined our Keck/NIRC2 relative astrometry of LSPM J1314+1320AB with VLBA absolute astrometry from Paper I to perform a joint analysis of the orbit and parallax of the system. The VLBA observations only detect one component of the binary at all epochs, and we identify it as the secondary component LSPM J1314+1320B (defined as the component that is fainter in our $J$ - and $K$-band Keck data). This is because the orbital motion seen in the VLBA data is to the northeast over 2013-2014, and our Keck astrometry over the same time period indicates that the secondary was moving to the northeast relative to the primary. In addition to our Keck astrometry here, we also used results from Law et al. (2006) who measured a binary separation of $130 \pm 20$ mas and PA of $46^{\circ} .0 \pm 2.0$ from their Lucky imaging data on 2005 Jun 15 UT. The combined data set contains a total of seven epochs of resolved astrometry spanning 10.03 years and nine epochs of absolute astrometry spanning 4.65 years.

Our astrometric model includes 15 total parameters, and 6 of these are orbit parameters shared between the Keck and VLBA data. The six common parameters are orbital period $(P)$, eccentricity $(e)$, inclination $(i)$, argument of periastron $(\omega)$, mean longitude at the reference epoch $\left(\lambda_{\text {ref }}\right)$, and PA of the ascending node $(\Omega)$. We defined the reference epoch for our model as $t_{\text {ref }}=2455197.5$ JD (i.e., calendar date 2010.0). The 
Table 2

MCMC Posteriors for the Orbit and Parallax of LSPM J1314+1320AB

\begin{tabular}{|c|c|c|c|c|}
\hline Property & Median $\pm 1 \sigma$ & Best Fit & $95.4 \%$ c.i. & Prior/Notes \\
\hline Orbital period $P$ (year) & $9.58_{-0.08}^{+0.07}$ & 9.58 & $9.45,9.74$ & $1 / P$ (log-flat) \\
\hline Eccentricity $e$ & $0.6011_{-0.0025}^{+0.0022}$ & 0.6014 & $0.5964,0.6060$ & uniform, $0 \leqslant e<1$ \\
\hline Inclination $i\left(^{\circ}\right)$ & $49.34_{-0.23}^{+0.28}$ & 49.19 & $48.77,49.82$ & $\sin (i), 0^{\circ}<i<180^{\circ}$ \\
\hline PA of the ascending node $\Omega\left({ }^{\circ}\right)$ & $60.4 \pm 0.4$ & 60.2 & $59.6,61.3$ & uniform \\
\hline$\alpha_{2010}-\operatorname{median}\left(\alpha_{2010}\right)$ (mas) & $0.0 \pm 0.4$ & 0.0 & $-0.8,0.8$ & uniform, median $\left(\alpha_{2010}\right)=198^{\circ} .5841023$ \\
\hline$\delta_{2010}-\operatorname{median}\left(\delta_{2010}\right)(\mathrm{mas})$ & $0.0 \pm 0.9$ & 0.0 & $-1.7,1.7$ & uniform, median $\left(\delta_{2010}\right)=+13.3330434$ \\
\hline Proper motion in R.A. $\mu_{\alpha \cos \delta}\left({\left.\operatorname{mas~} \mathrm{yr}^{-1}\right)}^{-1}\right.$ & $-247.99 \pm 0.10$ & -248.01 & $-248.19,-247.78$ & uniform \\
\hline Proper motion in decl. $\mu_{\delta}\left(\right.$ mas $\left.\mathrm{yr}^{-1}\right)$ & $-183.58 \pm 0.22$ & -183.64 & $-184.05,-183.17$ & uniform \\
\hline Parallax $\pi$ (mas) & $57.975 \pm 0.045$ & 57.988 & $57.856,58.082$ & $1 / \pi^{2}$ (uniform volume density) \\
\hline Total mass $M_{\text {tot }}\left(M_{\text {Jup }}\right)$ & $184.5 \pm 1.6$ & 183.7 & $181.1,187.6$ & $\cdots$ \\
\hline Primary mass $M_{1}\left(M_{\mathrm{Jup}}\right)$ & $92.8 \pm 0.6$ & 92.5 & $91.5,94.0$ & $\cdots$ \\
\hline Secondary mass $M_{2}\left(M_{\text {Jup }}\right)$ & $91.7 \pm 1.0$ & 91.2 & $89.6,93.8$ & $\cdots$ \\
\hline Mass ratio $q \equiv M_{2} / M_{1}$ & $0.989 \pm 0.007$ & 0.986 & $0.975,1.002$ & $\cdots$ \\
\hline Distance $d(\mathrm{pc})$ & $17.249 \pm 0.013$ & 17.245 & $17.217,17.284$ & $\cdots$ \\
\hline Semimajor axis $a(\mathrm{au})$ & $2.528 \pm 0.009$ & 2.525 & $2.512,2.548$ & $\cdots$ \\
\hline Time of periastron $T_{0}(\mathrm{JD})$ & $2456498.5_{-1.8}^{+1.7}$ & 2456498.8 & $2456495.0,2456502.0$ & $\cdots$ \\
\hline
\end{tabular}

semimajor axes of the primary and secondary components about their barycenter are denoted as $a_{1}$ and $a_{2}$, respectively. Since our Keck data measure relative orbital motion, the Keck semimajor axis parameter was $a=a_{1}+a_{2}$, while the VLBA semimajor axis parameter was simply $a_{2}$. The remaining parameters are only used to model the VLBA data: R.A. and decl. at the reference epoch $\left(\alpha_{2010}, \delta_{2010}\right)$; proper motion in R.A. and decl. $\left(\mu_{\alpha \cos \delta}, \mu_{\delta}\right)$; parallax $(\pi)$; and systematic error parameters in R.A. and decl. $\left(\sigma_{\alpha}, \sigma_{\delta}\right)$. These VLBA error parameters are needed to model systematic errors in the absolute astrometric calibration, which are expected to be comparable from epoch to epoch. In order to properly mitigate our model from preferring extremely large values of these error parameters, we penalized the logarithm of the likelihood by $\log \left(1 / \sigma^{2}\right)$, where $\sigma$ is the quadrature sum of both error parameters. The parallax factor at each epoch was calculated from the JPL ephemeris DE405 as described in Equations (1) and (2) of Dupuy \& Liu (2012).

As in our previous work on joint analysis of relative and absolute astrometry (Dupuy et al. 2015), we used the affine invariant ensemble sampler emcee v2.1.0 (ForemanMackey et al. 2013) to perform our Markov Chain Monte Carlo (MCMC) analysis. We used $10^{3}$ walkers (chains) with $10^{5}$ steps each, saving every 500th step for use in our analysis and removing the first $10 \%$ of all walkers as the burn-in portion. Table 2 summarizes the resulting posterior distributions and the priors on all of our parameters. We report both the best-fit parameters and the credible intervals that encompass $68.3 \%$ and $95.4 \%$ of each parameter's posterior values. The best-fit parameters give a total $\chi^{2}=17.8$ for 19 dof, $p$ $\left(\chi^{2}\right)=0.53$, including both the Keck data and the VLBA data with the systematic errors added in quadrature to the nominal VLBA measurement errors. Figure 2 displays this best-fit orbit and parallax solution alongside both the Keck and VLBA astrometry.

Table 2 also gives credible intervals and best-fit values for a number of additional parameters that can be derived directly from our fitted parameters, e.g., the distance computed from the absolute VLBA parallax $(d=1 / \pi)$. Most notably, we compute a precise total system mass from the Keck orbit and VLBA parallax, $M_{\mathrm{tot}}=(a / \pi)^{3} P^{-2}$, and individual masses for the components by combining the VLBA and Keck orbits $\left(M_{1}=\frac{a_{2}}{a} M_{\mathrm{tot}}, \quad M_{2}=\frac{a-a_{2}}{a} M_{\mathrm{tot}}\right)$. Because of the very high precision of the VLBA parallax $\left(\sigma_{\pi} / \pi=7.8 \times 10^{-4}\right)$, the uncertainties in these masses are dominated by the uncertainty in the orbital period $\left(\sigma_{P} / P=7.7 \times 10^{-3}\right)$, total semimajor axis $\left(\sigma_{a} / a=3.5 \times 10^{-3}\right)$, and secondary semimajor axis $\left(\sigma_{a_{2}} / a_{2}=4.5 \times 10^{-3}\right)$. Accounting for covariances between these parameters, the derived primary mass is the most precise $\left(\sigma_{M_{1}} / M_{1}=6.8 \times 10^{-3}\right)$, followed by the total mass $\left(\sigma_{M_{\mathrm{tot}}} / M_{\mathrm{tot}}\right.$ $\left.=8.7 \times 10^{-3}\right)$ and secondary mass $\left(\sigma_{M_{2}} / M_{2}=1.1 \times 10^{-2}\right)$. The mass ratio $\left(q \equiv M_{2} / M_{1}=\frac{a}{a_{2}}-1=0.989 \pm 0.007\right)$ is consistent with the radio-emitting secondary that is fainter in the NIR being the less massive component, and $95.4 \%$ of our MCMC posterior values having $q<1$. The derived primary and secondary masses are $92.8 \pm 0.6 M_{\text {Jup }}$ $\left(0.0885 \pm 0.0006 M_{\odot}\right) \quad$ and $\quad 91.7 \pm 1.0 M_{\text {Jup }}(0.0875 \pm$ $\left.0.0010 M_{\odot}\right)$, respectively. These individual masses are very nearly equal, within $2 \%$ of each other at $90 \%$ confidence. For additional discussion of the parallax and proper motion determined from this joint analysis see Paper I. 

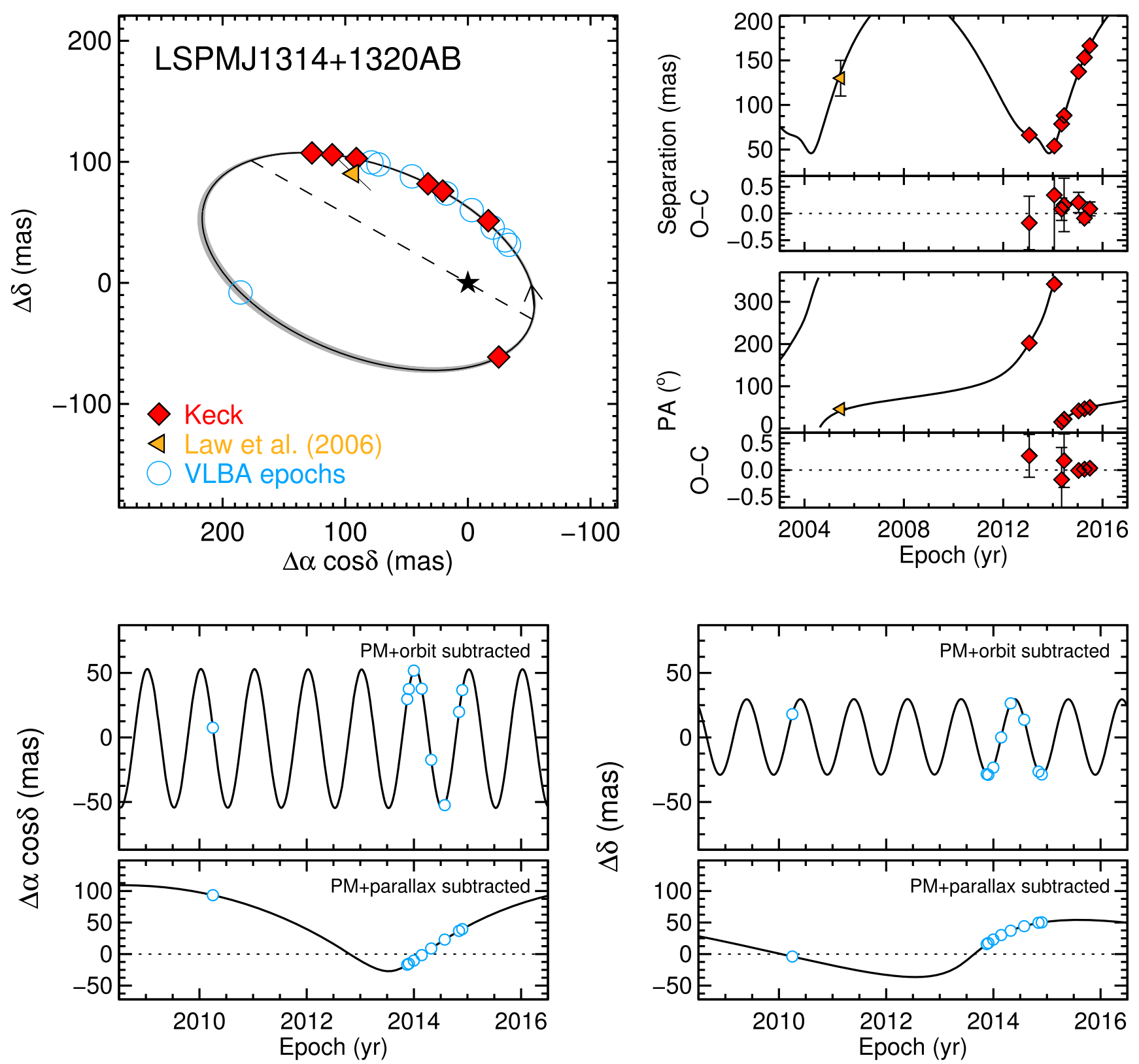

Figure 2. Top left: resolved relative astrometry (filled symbols) shown alongside the best-fit orbit (thick black line) and 100 randomly drawn orbits from our MCMC chain (thin gray lines). The plotting symbols typically are larger than the error bars. Open blue circles indicate the epochs of VLBA astrometry of the secondary from Paper I. Top right: our relative astrometry shown as a function of time (top sub-panels) and after subtracting the best-fit orbit solution (bottom sub-panels). Bottom: VLBA astrometry of the secondary from Paper I. Top panels show the data with the proper motion and orbital motion subtracted in order to display the best-fit parallax solution (thick black line). Bottom panels show the orbit of the secondary component after subtracting the best-fit parallax and proper motion.

\subsection{Other Empirically Determined Properties}

Our new AO imaging allows us to compute resolved photometry for the two components of LSPM J1314+1320AB. Our $J$ - and $K$-band flux ratios $(\Delta J=0.08 \pm 0.04 \mathrm{mag}$, $\Delta K=0.071 \pm 0.013 \mathrm{mag}$ ) are consistent with and more precise than the values of $\Delta J=0.10 \pm 0.11 \mathrm{mag}$ and $\Delta K_{S}=0.10 \pm 0.21 \mathrm{mag}$ from Schlieder et al. (2014). Using our $\Delta J$ and $\Delta K$ values and their $H$-band flux ratio, $\Delta H=0.03 \pm 0.06 \mathrm{mag}$, we computed resolved JHK photometry for the components of LSPM J1314+1320AB. For the integrated-light photometry, we used the 2MASS Point Source Catalog (Cutri et al. 2003) along with 2MASS-to-MKO photometric system conversions calculated from our SpeX spectrum. We list the resulting resolved and integrated-light photometry on both the MKO and 2MASS photometric systems in Table 3.

In order to compute the bolometric flux of LSPM J1314 $+1320 \mathrm{AB}$ in integrated light, we combined and absolutely calibrated our optical and NIR spectra following the method outlined in Mann et al. (2015). Briefly, we first collected published photometry from 2MASS, SDSS (Ahn et al. 2012), the AAVSO All-sky Photometric Survey (Henden et al. 2012), and the Wide-field Infrared Survey Explorer (WISE; Wright et al. 2010). We converted this photometry to fluxes using the relevant zero-points, and we also calculated synthetic fluxes from our spectra using the corresponding filter profiles (Cohen et al. 2003; Jarrett et al. 2011; Mann \& von Braun 2015). At this point, we noticed that the SDSS $i$-band measurement was 
Table 3

Properties of LSPM J1314+1320AB

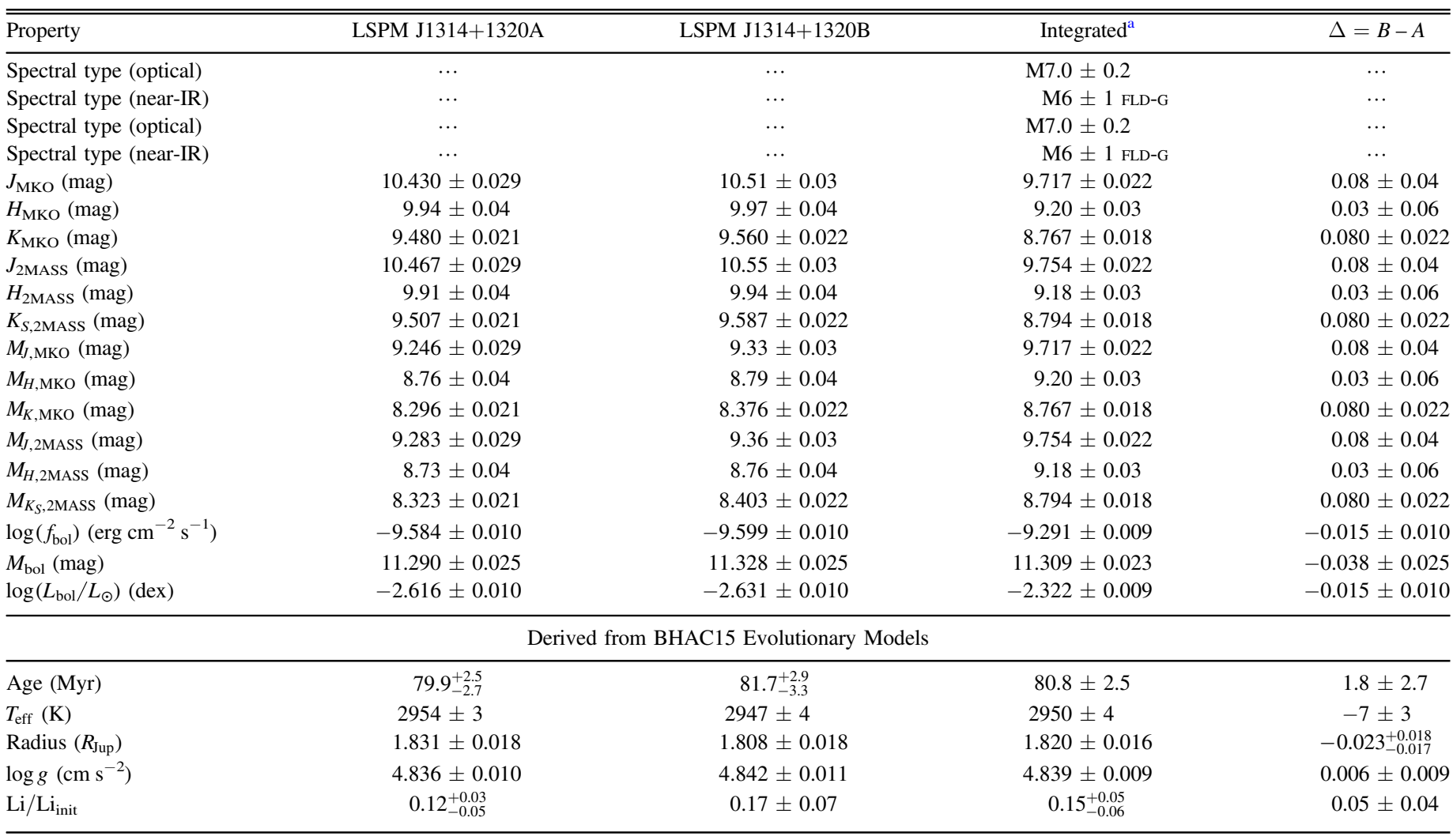

Note.

${ }^{a}$ Directly measured properties have their integrated-light values given. For model-derived properties, we report the mean of individually derived values for LSPM J1314+1320A and LSPM J1314+1320B.

highly discrepant ( $\approx 3-4$ mag) with our spectrum and with the surrounding photometry, so we excluded it from our analysis. Taking account of both random and correlated errors (flux calibration and optical variability), we scaled the optical and NIR spectra to match the photometry and to match each other in the region overlapping between SNIFS and SpeX $(0.80-0.95 \mu \mathrm{m})$. We replaced regions of high telluric contamination and those not covered by our spectra with a best-fit atmospheric model from the BT-Settl grid (Allard et al. 2011, 2012), where the best-fit here was $T_{\text {eff }}=2800 \mathrm{~K}$ and $\log g=4.5$ dex. The final calibrated and combined spectrum is shown in Figure 3. We calculated the bolometric flux $\left(f_{\text {bol }}\right)$ by integrating over the combined spectrum, accounting for errors in the flux calibration, optical variability of $1.5 \%$ (McLean et al. 2011; Williams et al. 2015), and errors introduced by replacing regions of spectra with a BT-Settl model. This yielded $f_{\text {bol }}=(5.12 \pm 0.10) \times 10^{-10} \mathrm{erg} \mathrm{s}^{-1} \mathrm{~cm}^{-2}$.

We apportioned this integrated-light bolometric flux to the individual components by deriving a bolometric flux ratio from our $K$-band flux ratio. We examined BT-Settl atmosphere models with $T_{\text {eff }}=2700-2900 \mathrm{~K}$ and $\log g=4.5-5.0 \mathrm{dex}$. Relative to the best-fit $T_{\text {eff }}=2800 \mathrm{~K}$ and $\log g=4.5 \mathrm{dex}$ model, the other five models had comparable or slightly larger amplitude $K$-band flux ratios spanning $\Delta K=-0.10$ mag to $0.12 \mathrm{mag}$ for hotter and cooler models, respectively. Among these models, the relationship between their bolometric magnitude differences and $K$-band magnitude differences was consistently $\Delta M_{\mathrm{bol}}-\Delta K=-0.042 \pm 0.011 \mathrm{mag}$. In comparison to our integrated-light bolometric magnitude error of

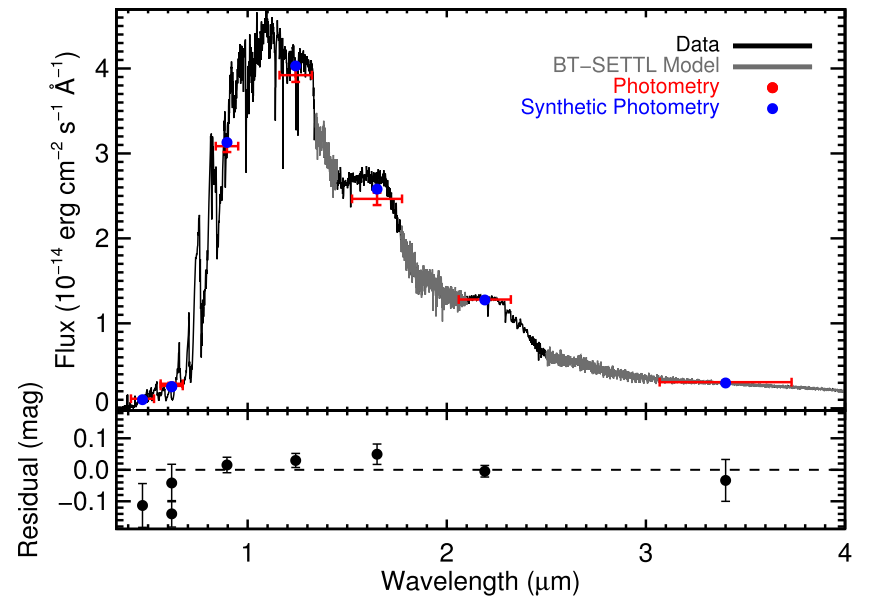

Figure 3. Flux-calibrated spectrum of LSPM J1314+1320AB from which we determine its integrated-light bolometric flux. Combined SNIFS+SpeX data are shown in black, and spectral regions replaced by models are shown in gray. Literature photometry is shown in red, with the horizontal bars indicating the width of the filter and vertical error bars representing combined measurement and zero point errors. Blue points indicate the corresponding synthetic fluxes derived from the spectrum. The bottom panel shows the photometry residuals. This plot is truncated at $4.0 \mu \mathrm{m}$ for display purposes, but we include all WISE photometry in our analysis.

$0.025 \mathrm{mag}$, the correction itself is small and the uncertainty in the correction is essentially negligible (though we do account for it in our analysis). Combining this correction factor with 


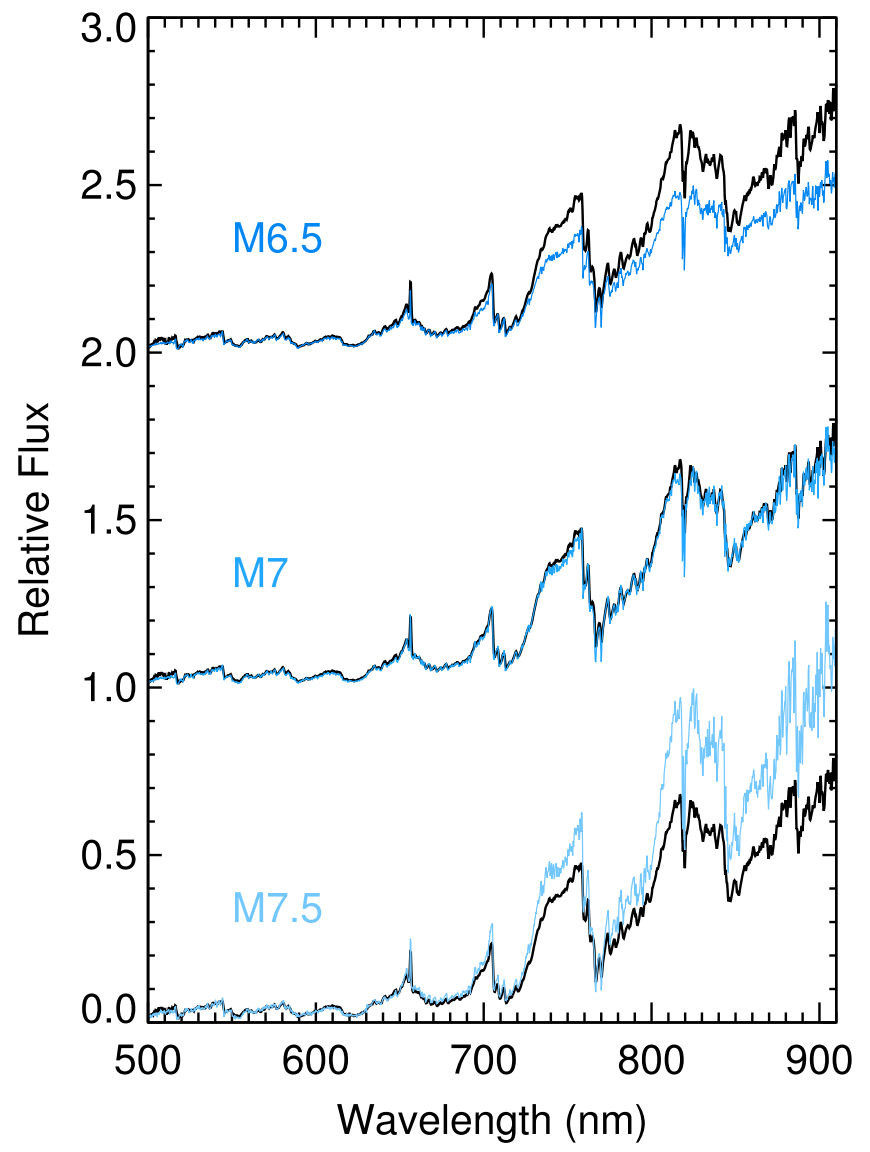

Figure 4. SNIFS integrated-light spectrum of LSPM J1314+1320AB shown in black compared to the M6.5, M7, and M7.5 spectral standards from Bochanski et al. (2007) shown in shades of blue. Interpolating these standards in a leastsquares fit, we determined a spectral type of M7.0 \pm 0.2 .

our $K$-band photometry, we find $\Delta \log \left(L_{\text {bol }}\right)=-0.015$ $\pm 0.010 \mathrm{dex}$, with the resultant individual luminosities given in Table 3.

\subsection{Spectral Type and Gravity Classification}

We determined the integrated-light spectral type of LSPM J1314+1320AB from our optical and NIR data. We compared our optical SNIFS spectrum to the M dwarf spectral library of Bochanski et al. (2007). To find a best-fit spectral type and uncertainty, we used the least-squares package MPFIT in IDL (Markwardt 2009). In performing this fit, we allowed the numerical spectral type to vary continuously, interpolating between standards to create the comparison spectra. We excluded wavelengths near $\mathrm{H} \alpha$, allowed for a small wavelength shift to account for radial velocity, and neglected extinction given the small distance. We found a best-fit spectral type for LSPM J1314+1320AB of M7.0 \pm 0.2 , and Figure 4 shows our data in comparison to the best-fit and adjacent spectral standards.

We also used our SpeX SXD spectrum to determine an NIR spectral type and gravity classification on the Allers \& Liu (2013a) system. We find a spectral type of M6 \pm 1 (Figure 5), consistent with our optical type and the previous determinations of M7.0 \pm 0.5 in the optical from Lépine et al. (2009) and M6.5 in the NIR from Schlieder et al. (2014). The gravity classification is determined from the amount of deviation in certain spectral features from field objects of similar spectral type. If most features are consistent with the field (i.e., having a score of zero), then the classification is FLD-G. If most features are deviant from the field indicating low gravity, then the classification is VL-G. Intermediate cases are given the classification INT-G. For LSPM J1314+1320, only three scores are used to determine the gravity, since the strength of VO absorption is not applicable at this spectral type. Based on the indices computed from our SXD spectrum (Table 4), we found that $\mathrm{FeH}$ was consisent with the field, most individual alkali lines were not strongly deviant from the field, and the $H$-band continuum was marginally inconsistent with the field. This results in a score of $0 \mathrm{n} 01$ and a formal classification of FLD-G. However, a closer examination of the alkali lines leads to a more nuanced interpretation. Of the four features used in the alkali score, the $\mathrm{K}_{\mathrm{I}}$ line at $1.169 \mu \mathrm{m}$ was consistently indicative of low gravity. Figure 6 shows all four alkali lines, and in fact all of them appear visually weaker than the field gravity M6 standard, consistent with the findings of Schlieder et al. (2014) from an independent spectrum. The other three are simply not weak enough to qualify as low gravity on the Allers \& Liu (2013a) system. Therefore, while the formal classification alone gives FLD-G, we note that LSPM J1314+1320 is more faithfully described as having a spectrum on the borderline between FLD-G and INT-G classifications.

Finally, we also used the $K$-band portion of our SpeX spectrum to determine the metallicity of LSPM J1314 $+1320 \mathrm{AB}$ in integrated light. Using the calibration of Mann et al. (2014) we found $[\mathrm{Fe} / \mathrm{H}]=0.04 \pm 0.08 \mathrm{dex}$, i.e., consistent with solar metallicity as assumed by theoretical models in the following analysis. We caution that this calibration was based on field objects and thus it might give a somewhat different value for $[\mathrm{Fe} / \mathrm{H}]$ than a relation based on low gravity dwarfs.

\subsection{Evolutionary Model-derived Properties}

Given our precisely determined individual masses and luminosities for the components of LSPM J1314+1320AB, we can uniquely infer other physical properties from evolutionary model tracks. We first interpolate the values of each physical parameter such as $T_{\text {eff }}$ from the BHAC 15 evolutionary tracks (Baraffe et al. 2015) onto uniform, 2-d grids in log(mass) and $\log \left(L_{\text {bol }}\right)$, using grid steps of 0.01 dex in both axes. We draw random, normally distributed values of the individual component's bolometric fluxes for each step in our MCMC chains. We then bilinearly interpolate each resultant pair of (mass, $L_{\text {bol }}$ ) from our chain on a given 2-d grid of parameter values to compute the posterior distributions of that parameter. This approach preserves covariances between input parameters, e.g., mass and $L_{\text {bol }}$ both depend on distance, when deriving parameters like $T_{\text {eff }}$ (e.g., Liu et al. 2008).

The resulting posterior distributions for model-derived values of age, $T_{\mathrm{eff}}$, radius, $\log g$, and fraction of lithium remaining $\left(\mathrm{Li} / \mathrm{Li}_{\text {init }}\right)$ are summarized in Table 3 . We find model-derived ages that are consistent with coevality at $0.7 \sigma$, giving a consensus age of $80.8 \pm 2.5 \mathrm{Myr}$. (This and other mean values given in Table 3 represent the posterior distribution of the mean of primary and secondary values calculated from each step of the chain.) Other model-derived parameters are comparably consistent between the two components, as expected given the very similar component masses and luminosities, with mean values of $T_{\text {eff }}=$ $2950 \pm 4 \mathrm{~K}, \log g=4.839 \pm 0.009 \mathrm{dex}, R=1.820 \pm 0.016$ 

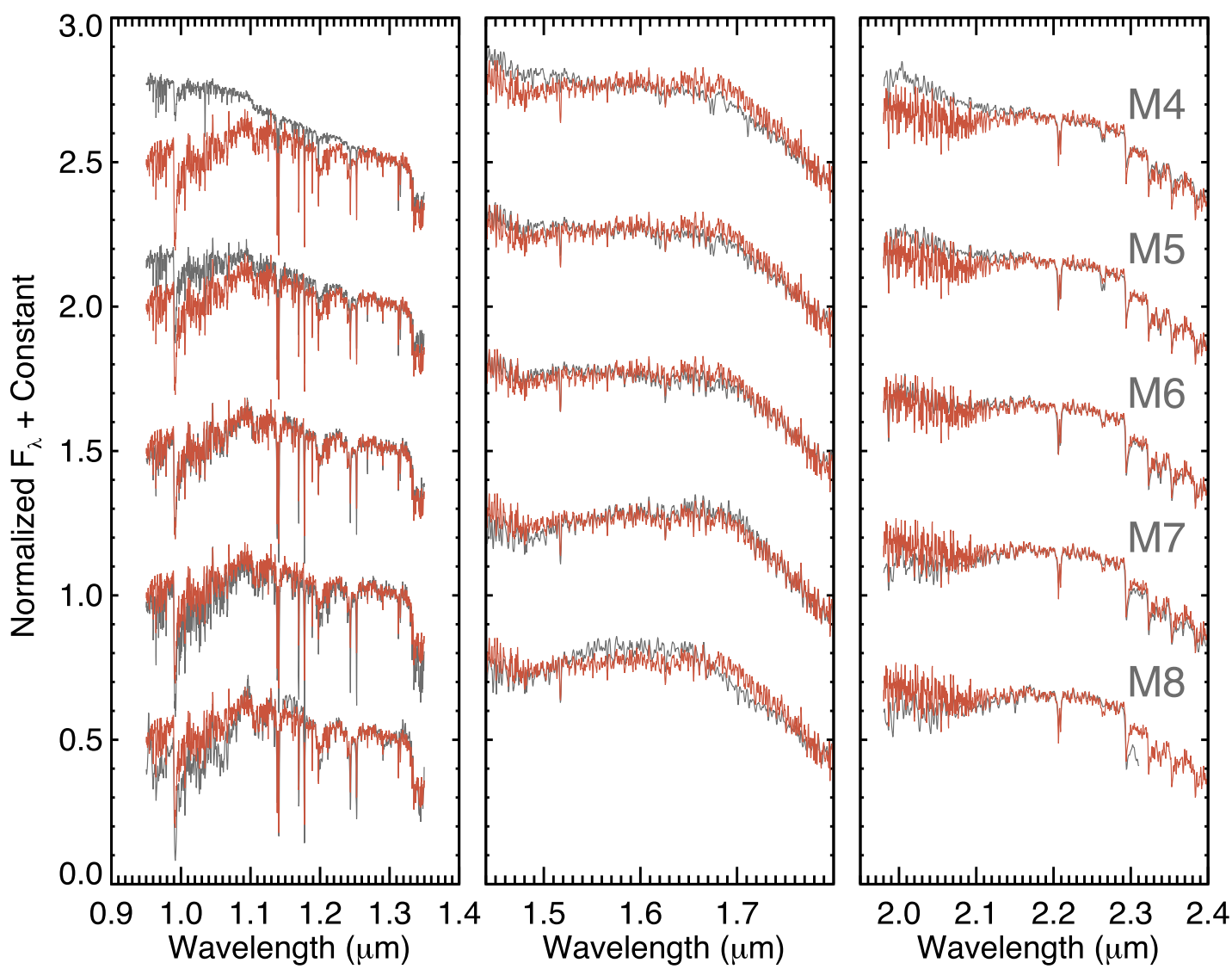

Figure 5. Integrated-light SpeX spectrum of LSPM J1314+1320AB (orange) compared to infrared spectral standards from Allers \& Liu (2013a, gray). The spectral type on this system is M6 \pm 1 .

Table 4

Gravity Classification Summary

\begin{tabular}{lc}
\hline \hline Name & Index/Score \\
\hline $\mathrm{FeH}_{z}$ & $1.1088 \pm 0.0012$ \\
$\mathrm{FeH}_{J}$ & $1.0722 \pm 0.0019$ \\
$\mathrm{VO}_{z}$ & $1.0010_{-0.0010}^{+0.0009}$ \\
$\mathrm{~K}_{J}$ & $1.0482 \pm 0.0006$ \\
$H$-cont & $0.9905 \pm 0.0006$ \\
$\mathrm{Na}_{\mathrm{I}}$ & $8.22 \pm 0.10$ \\
$\mathrm{~K}_{\mathrm{I}}(1.169 \mu \mathrm{m})$ & $2.10 \pm 0.10$ \\
$\mathrm{~K}_{\mathrm{I}}(1.177 \mu \mathrm{m})$ & $3.61_{-0.09}^{+0.10}$ \\
$\mathrm{~K}_{\mathrm{I}}(1.253 \mu \mathrm{m})$ & $2.63 \pm 0.08$ \\
Alkali Score & 1000 \\
Final Score & $0 \mathrm{n} 01$ \\
Gravity Classification & FLD-G \\
\hline
\end{tabular}

Note. The parts of the final score correspond, respectively, to lines of $\mathrm{FeH}(0)$, VO (n/a), alkali (0), and $H$-band continuum (1).

$R_{\mathrm{Jup}}$, and $\mathrm{Li} / \mathrm{Li}_{\text {init }}=0.15_{-0.06}^{+0.05}$. The very small formal uncertainties in our model-derived properties reflect the precision of the measured masses and luminosities projected onto the model grids; we do not attempt to include any systematic errors that could be associated with the models.

Evolutionary models indicate that the components of LSPM J1314+1320AB are well removed from the main sequence. For our measured masses of $0.0885 \pm 0.0006 M_{\odot}$ and $0.0875 \pm 0.0010 M_{\odot}, \quad$ BHAC15 models predict a main sequence luminosity of $\log \left(L_{\mathrm{bol}} / L_{\odot}\right)=-3.25 \mathrm{dex}$ attained by an age of $\approx 900$ Myr. Our measured luminosities, $\log \left(L_{\mathrm{bol}} / L_{\odot}\right)=-2.617 \pm 0.010 \mathrm{dex} \quad$ and $-2.629 \pm$ $0.010 \mathrm{dex}$, are a factor of $\approx 4 \times$ higher than the main-sequence value. The detection of lithium by Schlieder et al. (2014) in the combined light spectrum of these $\approx 0.09 M_{\odot}$ objects further supports the pre-main-sequence nature of this binary, as models predict lithium will be destroyed in objects of this mass hundreds of megayears before they reach the main sequence.

\section{DISCUSSION}

\subsection{Lithium Depletion and Age}

For very-low-mass stars $\left(\lesssim 0.1 M_{\odot}\right)$ and high-mass brown dwarfs, lithium is destroyed at a slow enough rate that it can be used to determine the ages of stellar associations up to at least $\sim 100 \mathrm{Myr}$. Higher mass objects destroy their primordial lithium at a faster rate, so at older ages lithium disappears from the spectra of progressively lower mass objects. Therefore, the brightest stars to display Li I (6708 $\AA$ ) absorption in a given cluster define an empirical boundary that can be used as a relative age scale between different clusters, and evolutionary models can be used to infer absolute ages of individual clusters based on the location of this lithium depletion boundary (e.g., Bildsten et al. 1997; Binks \& Jeffries 2014; Kraus et al. 2014). LSPM J1314+1320AB allows us to test these model predictions with stars of known mass for the first time at ages comparable to those of nearby open clusters.

Schlieder et al. (2014) measured a Li I (6708 А) pseudoequivalent width of $\mathrm{EW}=0.46 \AA$ from the integrated-light spectrum of LSPM J1314+1320AB. They noted that this is 

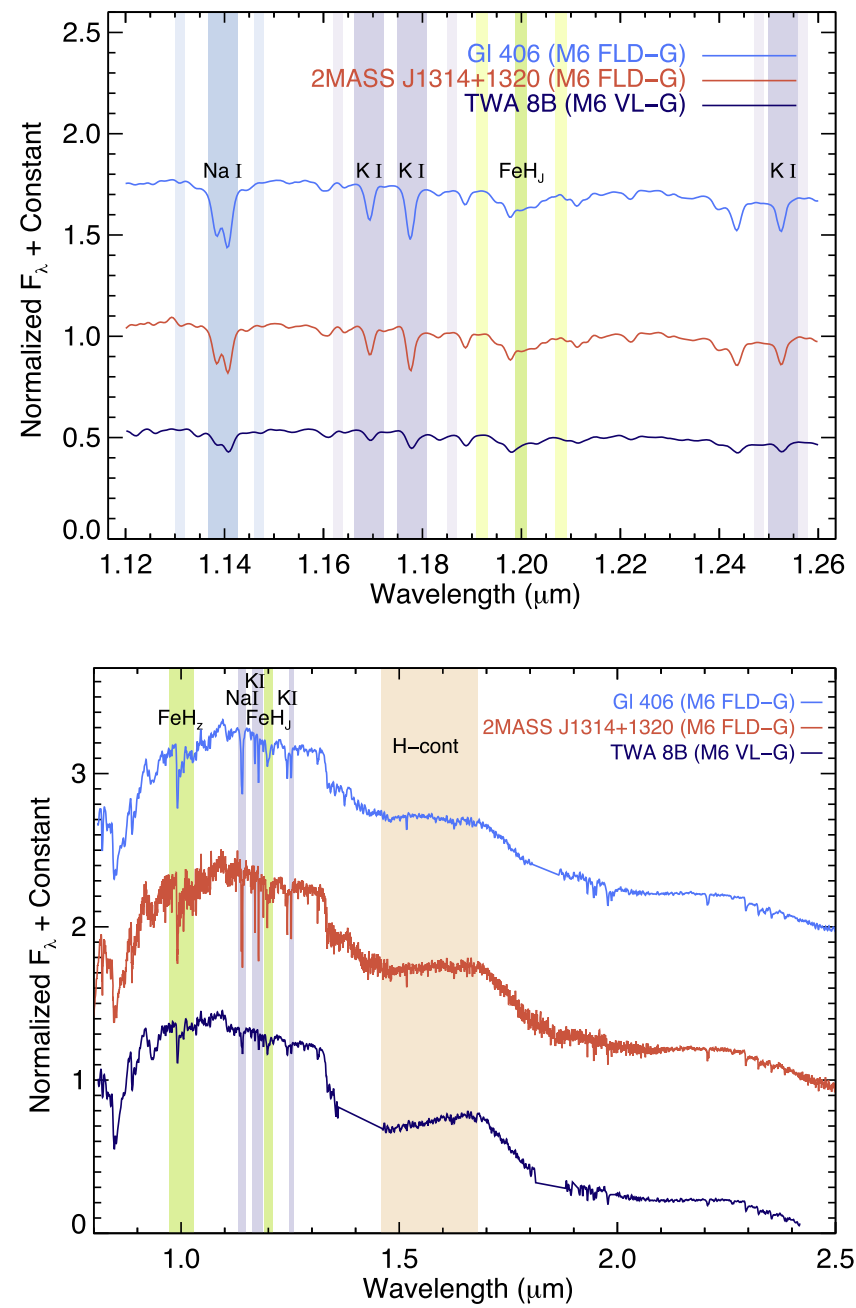

Figure 6. Top: a portion of our SpeX spectrum in $J$ band shown in comparison to M6 dwarfs with field gravity (FLD-G) and very low gravity (VL-G). Although the alkali lines visually appear intermediate between field- and low-gravity M6 dwarfs, a full analysis of all gravity scores from Allers \& Liu (2013a) yields a classification of FLD-G. LSPM J1314+1320AB is best described as having a spectrum on the borderline between FLD-G and INT-G classifications. Bottom: the full SpeX spectrum with other indices $(\mathrm{FeH}, \mathrm{VO}$, and $H$-band continuum shape) labeled.

consistent with measurements of comparable Pleiades late-M dwarfs, e.g., Stauffer et al. (1998) report three M7 dwarfs that all have $\mathrm{EW}=0.5-0.6 \AA$. Given our mass and luminosity measurements for each component, BHAC15 evolutionary models predict that the fraction of initial lithium remaining in the primary and secondary components is $0.12_{-0.05}^{+0.03}$ and $0.17 \pm 0.07$, respectively. Since the component masses are nearly equal, the predicted lithium depletion is correspondingly consistent within $1.2 \sigma$. The mean lithium fraction of the two components is predicted to be $\mathrm{Li} / \mathrm{Li}_{\text {init }}=0.15_{-0.06}^{+0.05}$.

An equivalent width measurement cannot be directly converted into lithium abundance. Palla et al. (2007) used a model dependent curve of growth approach to estimate the relationship between $\mathrm{Li}$ I pseudo-equivalent widths and lithium abundance, defined by $A(\mathrm{Li}) \equiv \log (N(\mathrm{Li}) / N(\mathrm{H}))+12$. If the lithium depletion level in the components of LSPM J1314 $+1320 \mathrm{AB}$ is $\approx 0.15$ as predicted by evolutionary models, and the initial cosmic lithium abundance is $A(\mathrm{Li}) \approx 3.3 \mathrm{dex}$ (Anders \& Grevesse 1989), then their present day abundance would be $A(\mathrm{Li}) \approx 2.5 \mathrm{dex}$. Over a range of
$T_{\text {eff }}=3100-3600 \mathrm{~K}$ at $\log g=4.5 \mathrm{dex}$, Palla et al. (2007) found that $A(\mathrm{Li})=2.5$ dex corresponds to $\mathrm{EW}=0.41-0.51 \AA$, which is consistent with the lithium detection from Schlieder et al. (2014). If this calibration from Palla et al. (2007) is accurate and applicable at the somewhat lower $T_{\text {eff }}$ here ( $\approx 3000 \mathrm{~K}$ according to evolutionary models; Section 3.4 ), then the detection of lithium absorption is fully consistent with the evolutionary model prediction that the components of LSPM J1314+1320AB have depleted most of their initial lithium supply.

As discussed in Section 3.4, models predict an age of $80.8 \pm 2.5 \mathrm{Myr}$ based on the mass and luminosity of the components of LSPM J1314+1320AB. We can place this in the context of the relative age scale provided by nearby open clusters with well determined lithium depletion boundaries. For the Pleiades, the boundary is at $M_{K_{S}}=8.78 \pm 0.05 \mathrm{mag}$ (Dahm 2015) using the VLBI parallax distance of Melis et al. (2014). The components of LSPM J1314+1320AB are 0.4-0.5 mag brighter than this (Table 3), implying that they must be significantly younger than the Pleiades in order to still possess lithium. Likewise for Blanco 1, Cargile et al. (2010) found a lithium depletion boundary of $M_{\mathrm{bol}}=11.99$ $\pm 0.30 \mathrm{mag}, \approx 0.7 \mathrm{mag}$ fainter than the components of LSPM J1314+1320AB, so they must also be younger than Blanco 1. The younger cluster $\alpha$ Persei has a lithium depletion boundary of $M_{\mathrm{bol}}=11.31 \mathrm{mag}$ (Barrado y Navascués et al. 2004), which is actually consistent within the errors for both components of LSPM J1314+1320AB. Therefore, we conclude that the age of LSPM J1314+1320AB must be consistent with or younger than that of $\alpha$ Per. Barrado $\mathrm{y}$ Navascués et al. (2004) report an age of $85 \pm 10 \mathrm{Myr}$ for $\alpha$ Per, and recent age determinations for the Pleiades and Blanco 1 are, respectively, $112 \pm 5 \mathrm{Myr}$ (Dahm 2015) and $132 \pm 24$ Myr (Cargile et al. 2010). The age of LSPM J1314 $+1320 \mathrm{AB}$ that we derived from models using mass and luminosity $(80.8 \pm 2.5 \mathrm{Myr})$ is therefore consistent with the requirement from lithium for the system age to be equal to or younger than the age of $\alpha$ Per.

Finally, we compare the integrated-light color and resolved absolute magnitudes of LSPM J1314+1320AB to the cluster sequences of $\alpha$ Per and the Pleiades. Using our observed spectrum, we compute integrated-light apparent magnitudes on the Cousins system $\left(I_{\mathrm{C}}=11.87 \pm 0.05 \mathrm{mag}\right)$ and $\mathrm{SDSS}$ system $\left(i^{\prime}=12.83 \pm 0.04 \mathrm{mag}\right)$. These give integrated-light colors of $I_{C}-K_{S}=3.08 \pm 0.05 \mathrm{mag}$ and $i^{\prime}-K_{S}=4.04$ $\pm 0.04 \mathrm{mag}$. The $\alpha$ Per members from Barrado y Navascués et al. (2002) within $0.15 \mathrm{mag}$ of this color have apparent magnitudes of $K_{S}=14.1 \pm 0.3 \mathrm{mag}$, and assuming a distance of $172.4 \pm 2.7 \mathrm{pc}$ (van Leeuwen 2009) gives an absolute magnitude of $M_{K_{S}}=7.9 \pm 0.3 \mathrm{mag}$. This is somewhat brighter than, but consistent with, the absolute magnitudes of the LSPM J1314+1320AB components $\left(M_{K_{S}}=8.32 \pm 0.02 \mathrm{mag}\right.$ and $8.40 \pm 0.02 \mathrm{mag})$. Performing the same exercise for the DANCe sample of probable $(p>0.99)$ Pleiades members from Bouy et al. (2015) using our $i^{\prime}-K_{S}$ color gives $K_{S}=14.2 \pm 0.3 \mathrm{mag}$ and thereby $M_{K_{S}}=8.5 \pm 0.3 \mathrm{mag}$ assuming a Pleiades distance of $136.2 \pm 1.2 \mathrm{pc}$ (Melis et al. 2014). This is somewhat fainter than, but consistent with, the absolute magnitudes of the LSPM J1314+1320AB components. The scatter in these cluster sequences on the color-magnitude diagram $(\approx 0.3 \mathrm{mag})$ is relatively large compared to the change of $\approx 0.6 \mathrm{mag}$ in absolute magnitude 

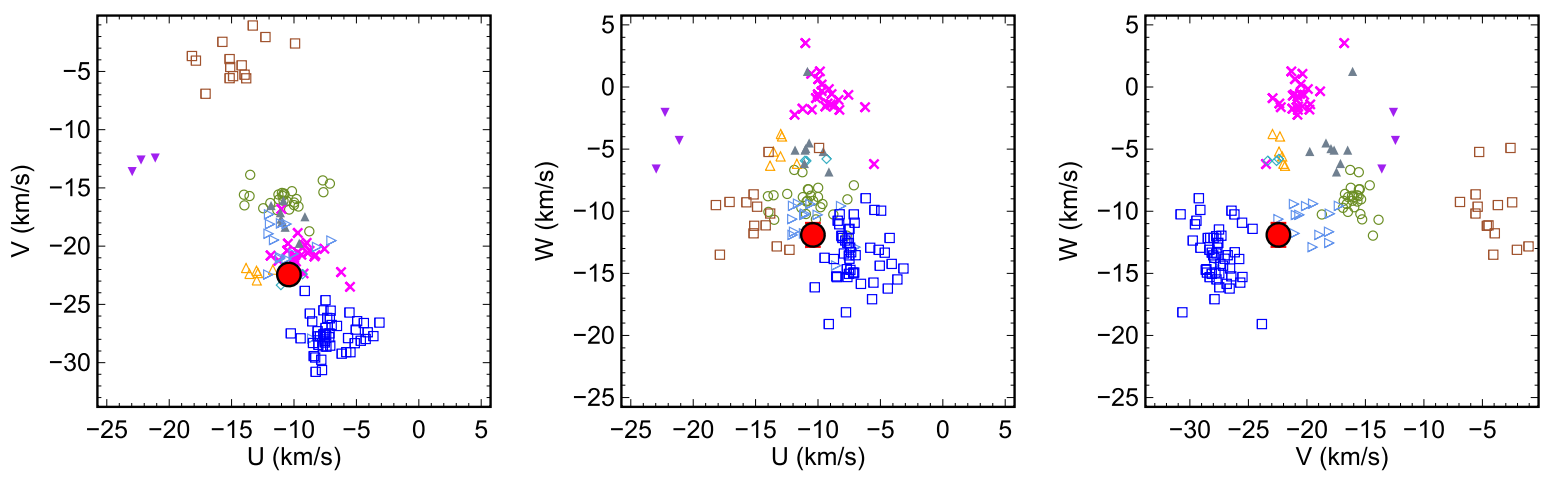

$\circ$ BPic $\times$ Tuc/Hor $\triangle$ Columba $\diamond$ Carina $\Delta$ TW Hya $\triangleright \eta$ Cha $\square$ Octans $\quad \nabla$ Argus $\quad \square$ AB Dor
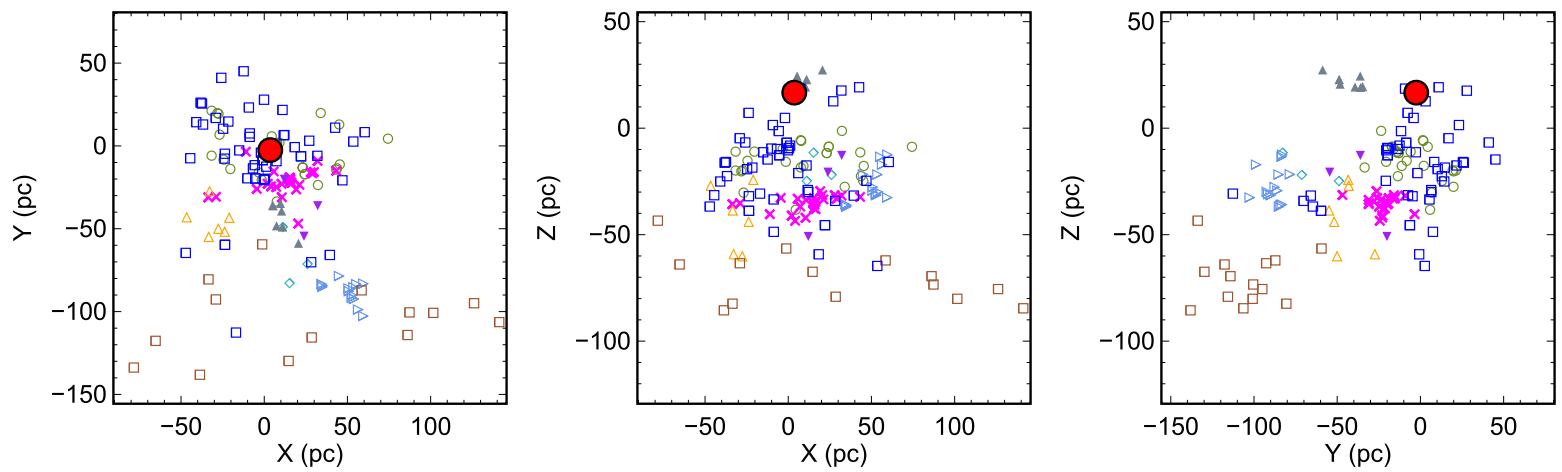

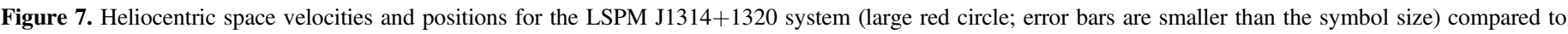

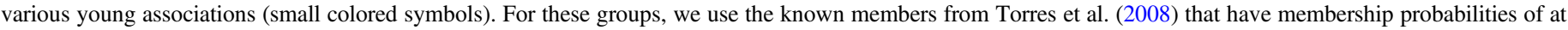

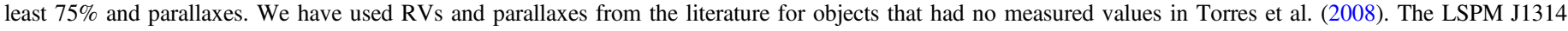
+1320 system does not seem to be associated with any known young moving groups.

from $\alpha$ Per to the Pleiades, which limits the discriminating power of this comparison. The components are somewhat fainter than expected for being as old or younger than $\alpha$ Per (0.4-0.5 mag), but the effect is not significant. We therefore conclude that the location of the components of LSPM J1314 $+1320 \mathrm{AB}$ on the color-magnitude diagram is consistent with the more precise constraints on age from the lithium depletion boundary comparison, being comparable in age to $\alpha$ Per and younger than the Pleiades.

\subsection{Membership Assessment}

Combining our proper motion and parallax with the published radial velocity of the LSPM J1314+1320AB system allows us to derive its space motion and thereby assess potential membership in known associations of young stars. Schlieder et al. (2014) reported spectrally resolved radial velocities of the two components of LSPM J1314+1320AB from which they computed a system velocity of $-10.4 \pm 1.0 \mathrm{~km} \mathrm{~s}^{-1}$ under the assumption that the two components are equal in mass. Our astrometric mass ratio of $q=0.989 \pm 0.007$ now validates this assumption within their measurement uncertainty and thereby their reported system velocity.

We derive a space motion of $(U, V, W)=(-10.4 \pm 0.21$, $-22.27 \pm 0.15,-11.9 \pm 1.0) \mathrm{km} \mathrm{s}^{-1}$ and plot this vector alongside various known young associations in Figure 7. There are no clear visual associations, except perhaps with $\eta$ Cha in $U V W$, but LSPM J1314+1320 is very far from $\eta$ Cha in $X Y Z$. Using the BANYAN II web tool (v1.4; Malo et al. 2013;
Gagné et al. 2014), we find a 99.98\% membership probability in the young field population assuming the age of the system is $<1$ Gyr. Therefore, according to BANYAN, LSPM J1314 +1320 is not likely a member of any of the seven young moving groups considered in their model (AB Dor, Argus, $\beta$ Pic, Carina, Columba, Tuc-Hor, and TWA).

We therefore conclude that the LSPM J1314+1320AB does not belong to any known young association, despite being unambiguously pre-main-sequence and located at only $17.25 \mathrm{pc}$. It is possible that LSPM J1314+1320AB belongs to an as yet unidentified $<100 \mathrm{Myr}$ association in the solar neighborhood, and if so this should be testable with the upcoming release of Gaia astrometry (de Bruijne 2012). If no new associations are found that match LSPM J1314+1320AB, then it will join the growing ranks of orphaned young objects in the solar neighborhood (e.g., Liu et al. 2016).

\subsection{Radius and Effective Temperature}

In principle, the rotation period $\left(P_{*}\right)$ and $v \sin \left(i_{*}\right)$ measurements from McLean et al. (2011) and Williams et al. (2015) provide an empirical constraint on the minimum stellar radius, $R \sin \left(i_{*}\right){ }^{6}$ In practice, such a calculation is complicated by the fact that their measurements are made in integrated light, but for the sake of argument we will assume that they both

\footnotetext{
6 We use the notation $P_{*}$ and $i_{*}$ here to indicate the stellar rotation period and the inclination of the stellar rotation axis with respect to the plane of the sky, respectively, since we have already used $P$ and $i$ for the binary orbit's period and inclination.
} 


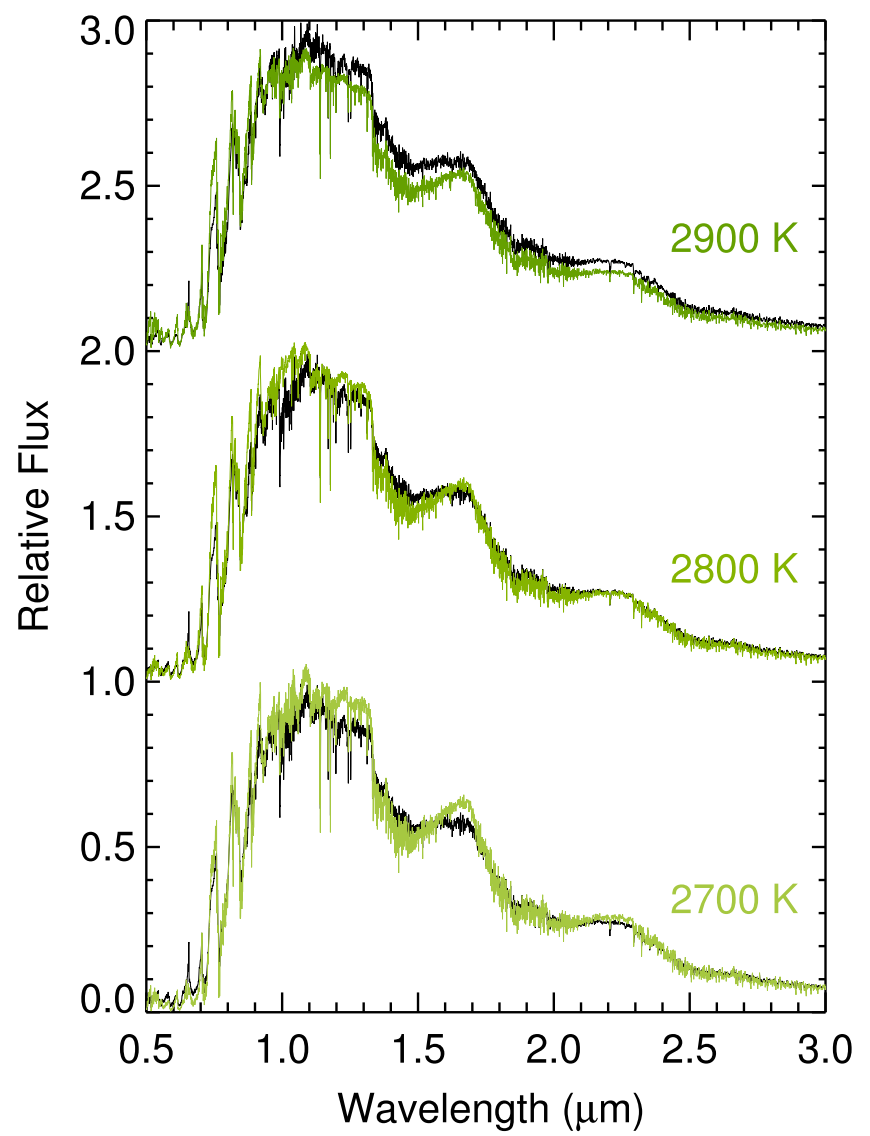

Figure 8. Our combined SNIFS+SpeX integrated-light spectrum of LSPM J1314+1320AB shown in black compared to BT-Settl model atmosphere spectra that all have surface gravity $\log g=4.5$ dex shown in shades of green. We fitted a full grid of BT-Settl model spectra to our data, and the bestfit spectrum had $T_{\text {eff }}=2800 \mathrm{~K}$ and $\log g=4.5 \mathrm{dex}$.

correspond to one of the two components of LSPM J1314 $+1320 \mathrm{AB}$. McLean et al. (2011) reported a rotation period of $3.89 \pm 0.05 \mathrm{hr}$ from multi-epoch VLA observations and a $v \sin \left(i_{*}\right)=45 \pm 5 \mathrm{~km} \mathrm{~s}^{-1}$ from optical spectroscopy. In optical photometric monitoring, Williams et al. (2015) find two distinct rotation periods of $3.7859 \pm 0.0001 \mathrm{hr}$ and $3.7130 \pm 0.0002 \mathrm{hr}$ and conclude that the cause of the two very similar, but distinct, periods is not clear. Assuming $45 \pm 5 \mathrm{~km} \mathrm{~s}^{-1}$ and $3.8 \mathrm{hr}$ gives $R \sin \left(i_{*}\right)=1.37 \pm 0.15 R_{\mathrm{Jup}}$. This is consistent with the model-derived average $R=1.820 \pm 0.016 R_{\mathrm{Jup}}$, which would correspond to $i_{*}=49 \pm 8^{\circ}$. Interestingly, this value is in good agreement with the measured orbital inclination $i=49.34_{-0.23}^{+0.28 \circ}$, which would be consistent with a stellar spin axis aligned with the orbital plane. However, it is also possible that the true radius is smaller or larger than predicted by models, corresponding to $i_{*}>49^{\circ}$ or $i_{*}<49^{\circ}$, respectively.

The BHAC15 evolutionary models that we have used to derive stellar parameters like radius and $T_{\text {eff }}$ employ BT-Settl model atmospheres as their boundary conditions for the surfaces of stars. Therefore, we can test for consistency between the value of $T_{\text {eff }}$ derived from BHAC15 and an independent estimate based on the spectrum of LSPM J1314 +1320 AB. Direct fitting of our combined optical and NIR spectrum with BT-Settl models yields $T_{\text {eff }}=2800 \mathrm{~K}$ and $\log g=4.5 \mathrm{dex}$ (Figure 8), but these values necessarily lack in precision due to the somewhat coarse $(100 \mathrm{~K}, 0.5 \mathrm{dex})$ model grid steps. The spectral type- $T_{\text {eff }}$ scale of Herczeg \& Hillenbrand (2014), which is based on BT-Settl model atmospheres, gives $T_{\text {eff }}=2770 \mathrm{~K}$ for a spectral type of M7, in good agreement with the direct fitting. Therefore, we find the BT-Settl models give $\approx 180 \mathrm{~K}$ cooler values for $T_{\text {eff }}$ than the average evolutionary model-derived value of $2950 \pm 4 \mathrm{~K}{ }^{7}$

In the absence of additional information, it is equally possible that this $T_{\text {eff }}$ discrepancy could be caused by systematic errors in either or both of the evolutionary and atmosphere models. Indeed, the BT-Settl models do not fit the overall spectrum with high accuracy (Figure 8), implying that any temperature based on these models will harbor some systematic error. Observations of other pre-main-sequence binaries at younger ages show discrepancies consistent with the $180 \mathrm{~K}$ difference here. For example, Rizzuto et al. (2016) found that for two unequal-mass $\mathrm{M}$ dwarf binary systems in the 10Myr-old Upper Scorpius subgroup, the evolutionary modelderived temperatures were $100-300 \mathrm{~K}$ higher than model atmospheres. If the $T_{\text {eff }}$ discrepancy for LSPM J1314 $+1320 \mathrm{AB}$ were due to evolutionary models, then at fixed luminosity this would imply model radii that are too small by $13 \%$. Interestingly, there are other cases of pre-main-sequence M dwarfs for which such underpredicted model radii can explain observed discrepancies. Kraus et al. (2015) found that for the 10 Myr old M5 eclipsing binary UScoCTIO 5, multiple evolutionary models (including BHAC15) underpredicted the component radii by $10 \%-15 \%$ while simultaneously overpredicting $T_{\text {eff }}$ by $\approx 300 \mathrm{~K}$ and thereby predicting consistent luminosities. In addition, evolutionary models of low-mass stars that include the effects of magnetic fields predict larger radii due a slowing down of their contraction (e.g., Macdonald \& Mullan 2010; Malo et al. 2014; Feiden 2016a, 2016b).

Therefore, both observations and theory suggest that the dominant source of the $T_{\text {eff }}$ discrepancy we observe for LSPM J1314+1320AB is most likely due to evolutionary model radii, although we note that spectral type $-T_{\text {eff }}$ scale could still harbor systematic errors. LSPM J1314+1320AB is much older than other pre-main-sequence systems with dynamical mass measurements in nearby star-forming regions, implying that the same qualitative radius $/ T_{\text {eff }}$ problem with evolutionary models extends to ages of at least $\sim 80 \mathrm{Myr}$.

\subsection{Spectral Signatures of Low Gravity}

To our knowledge, this is the first mass measurement for an ultracool dwarf (spectral type $\gtrsim M 7$ ) with spectral signatures of lower surface gravity than typical field objects. In Section 3.3, we classified the integrated-light spectrum as FLD-G, but on the borderline of being INT-G, and both Schlieder et al. (2014) and we note the presence of spectral features indicative of lower surface gravity relative to field dwarfs of similar spectral type. Evolutionary models indicate that the surface gravities of the binary components are $\log g=4.83-4.87 \mathrm{dex}$, which is $0.42-0.46$ dex lower than the predicted main-sequence surface gravity of $5.29 \mathrm{dex}$ for a $0.09 M_{\odot}$ star. If the model radii are too small by $13 \%$ as suggested above, then the model-derived

\footnotetext{
The spectral type- $T_{\text {eff }}$ scale of Luhman et al. (2003) gives a somewhat hotter $T_{\text {eff }}=2880 \mathrm{~K}$ at M7, in better agreement with the BHAC15 $T_{\text {eff }}$. However, this could be due to the fact that the Luhman et al. (2003) scale was designed to match evolutionary model isochrones of an earlier generation of the BHAC15 models and thus does not provide a truly independent $T_{\text {eff }}$ as needed for our consistency check. Moreover, using a spectral type- $T_{\text {eff }}$ scale intended for higher gravity field dwarfs (e.g., Rajpurohit et al. 2013) results in an even cooler, more inconsistent $T_{\text {eff }}$.
} 
gravities would be 0.1 dex lower. Overall, this implies that the borderline between FLD-G and INT-G designations for late-M dwarfs corresponds to a surface gravity $\approx 0.5$ dex lower than field objects.

While the LSPM J1314+1320AB system itself does not belong to an association of independently determined age, numerous other ultracool dwarfs with gravity classifications do. This allows us to check for consistency of spectral behavior between LSPM J1314+1320AB and other young late-M dwarfs of known age. In the $\mathrm{AB}$ Dor moving group (125 Myr), Aller et al. (2016) list 15 bona fide or strong candidate members. Of these, all but one object have gravity classifications of INT-G or VL-G, with only 2MASS J03264225-2102057 (L5) classified as FLD-G (but possessing some visual signs of youth, like LSPM J1314 $+1320 \mathrm{AB}$ but at a much later type). Of 17 possible candidate members none are classified FLD-G. Moreover, Allers \& Liu (2013b) report gravity classifications for eight ultracool dwarfs in the Pleiades; none were FLD-G, and the two M7 dwarfs PPl 1 and Teide 1 were INT-G and VL-G, respectively. These comparisons imply that spectral signatures leading to INT-G and VL-G classifications are typically still quite entrenched at ages significantly older than LSPM J1314+1320AB. At even younger ages, e.g., Tuc-Hor (50 Myr) and $\beta$ Pic (23 Myr), no known members are classified as FLD-G either (e.g., see compilation of Liu et al. 2016). Therefore, the fact that LSPM J1314+1320AB has a gravity classification of FLD-G is at odds with its model-derived age of $80.8 \pm 2.5 \mathrm{Myr}$ and that our lithium analysis that empirically places the system at a significantly younger age than the Pleiades.

We suggest a few possible explanations for the fact that LSPM J1314+1320AB shows less distinct evidence for low gravity than older ultracool dwarfs. Perhaps it is not that uncommon for late-M dwarfs to have more muted gravity signatures than L dwarfs, and the existing samples of ultracool dwarfs are too sparse to detect this yet. As noted by Allers \& Liu (2013b), the currently available infrared spectra of Pleiades late-M dwarfs are of much lower $\mathrm{S} / \mathrm{N}$ than was used to define the gravity classification system, so higher quality spectra may result in somewhat different, higher gravity, classifications. The classification of LSPM J1314+1320AB is done in integrated light, so perhaps the gravity signatures are somehow obscured in combined light. Allers \& Liu (2013b) tested such an idea, mostly using VL-G templates for components, and found that it is quite rare for two components to be classified as lower gravity in combined light. Finally, maybe some third parameter is at work, e.g., metallicity or rotation, causing the gravity classification to deviate slightly from other young ultracool dwarfs. Our spectrum shows no signs of unusual metallicity, and it also seems unlikely that such a young object would differ substantially in composition from other young associations in the solar neighborhood (e.g., Santos et al. 2008).

\subsection{H-R Diagram Test}

Pre-main-sequence stellar models are commonly used to infer masses by placing objects on the H-R diagram (e.g., Luhman et al. 1998; Preibisch et al. 2002; Da Rio et al. 2012). To test the accuracy of masses derived from models in this way, we used the effective temperatures and luminosities of LSPM J1314+1320AB to derive mass and age. Given that the masses and luminosities of the components are nearly equal, we simply consider the average integrated-light properties for

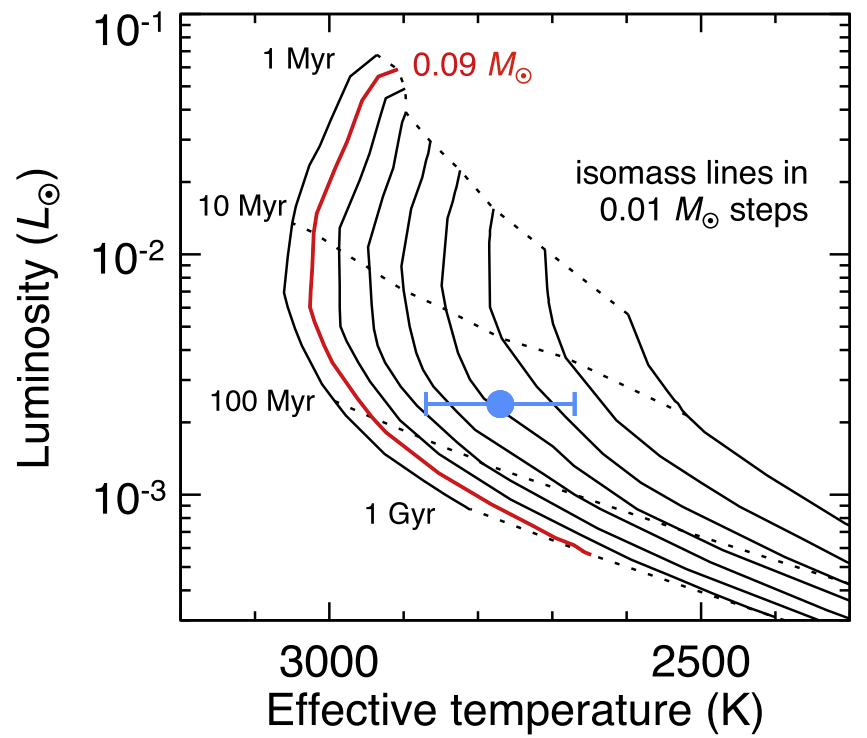

Figure 9. H-R diagram showing the mean luminosity of the LSPM J1314 $+1320 \mathrm{AB}$ components and the $T_{\text {eff }}$ determined from the integrated-light spectral type of M7.0 (blue data point) compared to evolutionary model tracks. BHAC15 isomass tracks are shown in steps of $0.01 M_{\odot}$ with the $0.09 M_{\odot}$ track highlighted in red since this is consistent within $<3 \%$ of both measured individual masses. Isochrones from $1 \mathrm{Myr}$ to $1 \mathrm{Gyr}$ are indicated by dotted lines. The $T_{\text {eff }}$ used for LSPM J1314+1320AB here is calibrated off of the BTSettl model atmospheres, the same used for boundary conditions in the BHAC15 evolutionary models. When we use this $T_{\text {eff }}$ and luminosity to infer properties from BHAC15 models, we find a mass $46_{-19}^{+16} \%(2.0 \sigma)$ lower than we measured dynamically. This discrepancy indicates either large errors in spectral type- $T_{\text {eff }}$ relations $(\approx 180 \mathrm{~K})$ or systematic errors in evolutionary models (e.g., $13 \%$ in radius). In either case, this result suggests that masses inferred for young stars from the H-R diagram will harbor large systematic errors, and young stars may be confused for young brown dwarfs.

this test. As mentioned above, the spectral type- $T_{\text {eff }}$ scale for young objects from Herczeg \& Hillenbrand (2014) gives $T_{\text {eff }}=2770 \mathrm{~K}$, and they estimate a systematic error of $100 \mathrm{~K}$ for their $T_{\text {eff }}$ scale, which we adopt here. The mean measured luminosity of LSPM J1314+1320AB is $\log \left(L_{\mathrm{bol}} / L_{\odot}\right)=-2.623 \pm 0.010$ dex. Figure 9 shows these values of $T_{\text {eff }}$ and $L_{\text {bol }}$ compared to BHAC15 evolutionary model tracks.

For this test, we interpolated model tracks on a uniform grid of $\log \left(L_{\mathrm{bol}}\right)$ and $\log \left(T_{\text {eff }}\right)$ in the same fashion as described above in Section 3.4. We found an H-R diagram derived average component mass of $50_{-20}^{+13} M_{\text {Jup }}$, age of $25_{-17}^{+10} \mathrm{Myr}$, and $\log g$ of $4.46_{-0.22}^{+0.20}$ dex. This would imply that LSPM J1314 $+1320 \mathrm{AB}$ is actually a pair of young brown dwarfs, due to the $\mathrm{H}-\mathrm{R}$ diagram derived mass being $46_{-19}^{+16} \%(2.0 \sigma)$ smaller than our directly measured component masses of $\approx 92 M_{\text {Jup }}$. The $\mathrm{H}-\mathrm{R}$ diagram age is also much smaller $(0.5 \pm 0.3 \mathrm{dex})$ than the age derived from the same models using mass and $L_{\mathrm{bol}}$. As expected, the mass and age posteriors derived from the H-R diagram are highly correlated, where lower masses correspond to younger ages.

The discrepancy between the H-R diagram derived mass and our dynamically measured masses suggests either large errors in the spectral type- $T_{\text {eff }}$ relations, which are calibrated using BT-Settl model atmospheres, systematic errors in the evolutionary models, or some combination of both things. As we discuss in Section 4.3, we suggest the dominant source of this discrepancy is that evolutionary model radii are underpredicted and $T_{\text {eff }}$ is thereby overpredicted at a given luminosity. 
Regardless of the cause of the discrepancy, this test case shows that masses derived from the H-R diagram can harbor large systematic errors. Unfortunately, this method is often the only practical option when attempting to infer masses of stars and brown dwarfs in young associations where age can be uncertain due to potential underlying age spreads. Therefore, the systematic error we have identified here will have significant implications for efforts to determine the low-mass end of the initial mass function, suggesting that young low-mass stars may be mistakenly identified as young brown dwarfs.

\section{CONCLUSIONS}

We present here individual dynamical masses for the components of LSPM J1314+1320AB, a pre-main-sequence binary located at a distance of only $17.249 \pm 0.013 \mathrm{pc}$. These masses and parallactic distance are made possible by a joint analysis of our resolved relative astrometry of the primary and secondary from Keck AO imaging and masking along with absolute astrometry from VLBA radio interferometry of the secondary (i.e., the component that is fainter in the optical and infrared). We also derive component luminosities using integrated-light spectroscopy and photometry and our resolved infrared photometry. The measured component masses of $92.8 \pm 0.6 M_{\mathrm{Jup}}\left(0.0885 \pm 0.0006 M_{\odot}\right)$ and $91.7 \pm 1.0 M_{\mathrm{Jup}}$ $\left(0.0875 \pm 0.0010 M_{\odot}\right)$ and luminosities of $\log \left(L_{\mathrm{bol}} / L_{\odot}\right)=$ $-2.616 \pm 0.010 \mathrm{dex}$ and $-2.631 \pm 0.010 \mathrm{dex}$, respectively, are consistent with being coeval at an age of $80.8 \pm 2.5 \mathrm{Myr}$ according to BHAC15 evolutionary models. Our precise masses and luminosities are largely thanks to a remarkably precise VLBA parallax $\left(\sigma_{\pi} / \pi=9 \times 10^{-4}\right.$; Paper I). We determine that LSPM J1314+1320AB is unambiguously in the pre-main-sequence phase of its evolution based on having lithium absorption and luminosities $\approx 4 \times$ higher than predicted for the main sequence at our measured masses. This combination of precise distance, masses, luminosities, and the detection of lithium by Schlieder et al. (2014) enables novel tests of pre-main-sequence models distinct from previous work on objects in star-forming regions with more uncertain distances.

1. Evolutionary models self-consistently predict luminosity and lithium depletion in this binary thereby passing the first test of the theoretical lithium depletion boundary using ultracool dwarfs of known mass. Models predict the components have lost $88_{-3}^{+5} \%$ and $83 \pm 7 \%$ of their initial lithium, leaving enough remaining that they are still expected to display lithium absorption. On the empirically defined relative cluster age scale, the presence of lithium and the component absolute magnitudes of LSPM J1314+1320AB imply an age consistent with or younger than $\alpha$ Persei and significantly younger than the Pleiades and Blanco 1.

2. We compare the effective temperature derived from evolutionary models given our mass and luminosity (component average $T_{\text {eff }}=2950 \pm 5 \mathrm{~K}$ ) to that derived from spectral type- $T_{\text {eff }}$ relations based on BT-Settl models $(2770 \pm 100 \mathrm{~K})$. The $180 \mathrm{~K}$ discrepancy in these values would correspond to a $13 \%$ radius error at fixed luminosity, which is similar to a problem observed for low-mass stars in the much younger Upper Scorpius starforming region. The eclipsing binary USco CTIO 5 shows that model radii are likely the dominant source of the $T_{\text {eff }}$ problem, while the radius and $T_{\text {eff }}$ errors cancel so that model luminosities are accurate. We suggest that the same physical cause is responsible for the $180 \mathrm{~K}$ discrepancy for LSPM J1314+1320AB, showing that this radius problem can extend to much older pre-mainsequence ages than previously recognized.

3. In a test that mimics the typical application of evolutionary models by observers, we used the $T_{\text {eff }}$ derived from spectral type and the average component luminosity to infer mass and age from evolutionary model tracks on the H-R diagram. The $T_{\mathrm{eff}}-L_{\mathrm{bol}}$ derived average component mass of $50_{-20}^{+13} M_{\text {Jup }}$ is much lower $\left(46_{-19}^{+16} \%, 2.0 \sigma\right)$ than we measure dynamically. This highlights the large systematic errors possible when inferring masses of low-mass stars and brown dwarfs at young ages and implies that some stars may be mistakenly identified as brown dwarfs when using the $\mathrm{H}-\mathrm{R}$ diagram.

4. The integrated-light spectrum of LSPM J1314+1320AB displays signatures of low surface gravity, though we formally classify it as FLD-G (on the borderline of INT-G) on the infrared Allers \& Liu (2013a) system. This is the first time dynamical masses have been measured for ultracool dwarfs with low-gravity spectral features. However, contrary to expectations, LSPM J1314 $+1320 \mathrm{AB}$ shows less distinct spectral signs of low gravity than ultracool dwarfs at older ages (Pleiades, AB Dor), which we are unable to explain.

LSPM J1314+1320AB provides a high-precision benchmark for pre-main-sequence models at a distance $\sim 10 \times$ closer than even the nearest star-forming regions. One major unresolved question is why only the secondary component is radio emitting, as discussed in detail in Paper I. Given that we have shown that the component masses are within $2 \%$ of each other for this coeval, co-compositional binary system, one likely explanation for the divergent behavior is a difference in the angular momentum evolution of the two components. Projected rotational velocities $(v \sin i)$ of other very-low-mass binaries have hinted at such differences in angular momentum evolution (e.g., Konopacky et al. 2012), but such measurements have yet to be obtained for LSPM J1314+1320AB. The components of LSPM J1314+1320AB are now the nearest, lowest mass pre-main-sequence stars with direct mass measurements. In the future, Gaia parallaxes combined with ongoing ground-based orbit monitoring efforts will make many more such tests of models possible for more distant binaries in star-forming regions. Gaia data could even help identify previously unrecognized associations of young stars in the solar neighborhood to which LSPM J1314+1320AB, currently not associated with any known group, may belong.

This work was supported by a NASA Keck PI Data Award, administered by the NASA Exoplanet Science Institute. We thank Will Best for assistance with some Keck/NIRC2 observations. It is a pleasure to thank Joel Aycock, Carolyn Jordan, Jason McIlroy, Luca Rizzi, Terry Stickel, Hien Tran, and the Keck Observatory staff for assistance with our Keck AO observations. We also thank P. K. G. Williams, Michael Ireland, Katelyn Allers, Joshua Schlieder, and Mark Reid for useful discussions. The anonymous referee provided a remarkably rapid and thoughtful review that helped refine our discussion. James R. A. Davenport for distributing his IDL 
implementation of the cubehelix color scheme (Green 2011). Our research has employed the 2MASS data products; NASA's Astrophysical Data System; data from the Wide-field Infrared Survey Explorer, which is a joint project of the University of California, Los Angeles, and the Jet Propulsion Laboratory/ California Institute of Technology, funded by NASA and curated by the NASA/IPAC Infrared Science Archive; and the SIMBAD database operated at CDS, Strasbourg, France. Finally, the authors wish to recognize and acknowledge the very significant cultural role and reverence that the summit of Maunakea has always had within the indigenous Hawaiian community. We are most fortunate to have the opportunity to conduct observations from this mountain.

Facilities: IRTF, Keck:II (NGS AO, NIRC2), UH:2.2 m.

\section{REFERENCES}

Ahn, C. P., Alexandroff, R., Allende Prieto, C., et al. 2012, ApJS, 203, 21 Aldering, G., Adam, G., Antilogus, P., et al. 2002, Proc. SPIE, 4836, 61 Allard, F., Homeier, D., \& Freytag, B. 2011, in ASP Conf. Ser. 448, 16th Cambridge Workshop on Cool Stars, Stellar Systems, and the Sun, ed. C. Johns-Krull, M. K. Browning, \& A. A. West (San Francisco, CA: ASP), 91

Allard, F., Homeier, D., \& Freytag, B. 2012, RSPTA, 370, 2765

Aller, K. M., Liu, M. C., Magnier, E. A., et al. 2016, ApJ, 821, 120

Allers, K. N., \& Liu, M. C. 2013a, ApJ, 772, 79

Allers, K. N., \& Liu, M. C. 2013b, MmSAI, 84, 1089

Anders, E., \& Grevesse, N. 1989, GeCoA, 53, 197

Bacon, R., Copin, Y., Monnet, G., et al. 2001, MNRAS, 326, 23

Baraffe, I., Homeier, D., Allard, F., \& Chabrier, G. 2015, A\&A, 577, A42

Barrado y Navascués, D., Bouvier, J., Stauffer, J. R., Lodieu, N., \& McCaughrean, M. J. 2002, A\&A, 395, 813

Barrado y Navascués, D., Stauffer, J. R., \& Jayawardhana, R. 2004, ApJ, 614, 386

Bastian, N., Covey, K. R., \& Meyer, M. R. 2010, ARA\&A, 48, 339

Bildsten, L., Brown, E. F., Matzner, C. D., \& Ushomirsky, G. 1997, ApJ, 482, 442

Binks, A. S., \& Jeffries, R. D. 2014, MNRAS, 438, L11

Bochanski, J. J., West, A. A., Hawley, S. L., \& Covey, K. R. 2007, AJ, 133,531

Bouy, H., Bertin, E., Sarro, L. M., et al. 2015, A\&A, 577, A148

Buton, C., Copin, Y., Aldering, G., et al. 2013, A\&A, 549, A8

Cargile, P. A., James, D. J., \& Jeffries, R. D. 2010, ApJL, 725, L111

Cohen, M., Wheaton, W. A., \& Megeath, S. T. 2003, AJ, 126, 1090

Cushing, M. C., Vacca, W. D., \& Rayner, J. T. 2004, PASP, 116, 362

Cutri, R. M., Skrutskie, M. F., van Dyk, S., et al. 2003, 2MASS All Sky Catalog of Point Sources, (The IRSA 2MASS All-Sky Point Source Catalog, NASA/IPAC Infrared Science Archive. http://irsa.ipac.caltech. edu/applications/Gator/)

Czekala, I., Andrews, S. M., Torres, G., et al. 2016, ApJ, 818, 156

Dahm, S. E. 2015, ApJ, 813, 108

Da Rio, N., Robberto, M., Hillenbrand, L. A., Henning, T., \& Stassun, K. G. 2012, ApJ, 748, 14

David, T. J., Hillenbrand, L. A., Cody, A. M., Carpenter, J. M., \& Howard, A. W. 2016, ApJ, 816, 21

de Bruijne, J. H. J. 2012, Ap\&SS, 341, 31

Diolaiti, E., Bendinelli, O., Bonaccini, D., et al. 2000, A\&AS, 147, 335

Dupuy, T. J., Kratter, K. M., Kraus, A. L., et al. 2016, ApJ, 817, 80

Dupuy, T. J., \& Liu, M. C. 2012, ApJS, 201, 19

Dupuy, T. J., Liu, M. C., Bowler, B. P., et al. 2010, ApJ, 721, 1725

Dupuy, T. J., Liu, M. C., \& Ireland, M. J. 2009a, ApJ, 692, 729

Dupuy, T. J., Liu, M. C., \& Ireland, M. J. 2009b, ApJ, 699, 168

Dupuy, T. J., Liu, M. C., Leggett, S. K., et al. 2015, ApJ, 805, 56

Feiden, G. A. 2016a, arXiv:1604.08036

Feiden, G. A. 2016b, in IAU Symp. 314, Young Stars and Planets Near the Sun, ed. J. H. Kastner, B. Stelzer, \& S. A. Metchev (Cambridge: Cambridge Univ. Press), 79

Forbrich, J., Dupuy, T. J., Reid, M. J., et al. 2016, ApJ, 827, 22

Foreman-Mackey, D., Hogg, D. W., Lang, D., \& Goodman, J. 2013, PASP, 125,306

Gagné, J., Lafrenière, D., Doyon, R., Malo, L., \& Artigau, É 2014, ApJ, 783, 121

Gaidos, E., Mann, A. W., Lépine, S., et al. 2014, MNRAS, 443, 2561
Gennaro, M., Prada Moroni, P. G., \& Tognelli, E. 2012, MNRAS, 420, 986 Green, D. A. 2011, BASI, 39, 289

Henden, A. A., Levine, S. E., Terrell, D., Smith, T. C., \& Welch, D. 2012, JAVSO, 40, 430

Herczeg, G. J., \& Hillenbrand, L. A. 2014, ApJ, 786, 97

Hillenbrand, L. A., \& White, R. J. 2004, ApJ, 604, 741

Ireland, M. J., Kraus, A., Martinache, F., Lloyd, J. P., \& Tuthill, P. G. 2008, ApJ, 678, 463

Ireland, M. J., \& Kraus, A. L. 2008, ApJL, 678, L59

Jarrett, T. H., Cohen, M., Masci, F., et al. 2011, ApJ, 735, 112

Konopacky, Q. M., Ghez, A. M., Fabrycky, D. C., et al. 2012, ApJ, 750, 79

Kraus, A. L., Cody, A. M., Covey, K. R., et al. 2015, ApJ, 807, 3

Kraus, A. L., Shkolnik, E. L., Allers, K. N., \& Liu, M. C. 2014, AJ, 147, 146 Lantz, B., Aldering, G., Antilogus, P., et al. 2004, Proc. SPIE, 5249, 146 Law, N. M., Hodgkin, S. T., \& Mackay, C. D. 2006, MNRAS, 368, 1917

Law, N. M., Hodgkin, S. T., \& Mackay, C. D. 2008, MNRAS, 384, 150 Lépine, S. 2005, AJ, 130, 1680

Lépine, S., \& Gaidos, E. 2011, AJ, 142, 138

Lépine, S., \& Shara, M. M. 2005, AJ, 129, 1483

Lépine, S., Thorstensen, J. R., Shara, M. M., \& Rich, R. M. 2009, AJ, 137, 4109

Liu, M. C., Dupuy, T. J., \& Allers, K. N. 2016, ApJ, submitted

Liu, M. C., Dupuy, T. J., \& Ireland, M. J. 2008, ApJ, 689, 436

Lodieu, N., Alonso, R., González Hernández, J. I., et al. 2015, A\&A, 584, A128

Luhman, K. L., Rieke, G. H., Lada, C. J., \& Lada, E. A. 1998, ApJ, 508, 347

Luhman, K. L., Stauffer, J. R., Muench, A. A., et al. 2003, ApJ, 593, 1093

Luyten, W. J. 1979, New Luyten Catalogue of Stars with Proper Motions Larger than Two Tenths of an Arcsecond (NLTT), University of Minnesota, Minneapolis (CDS-ViZier catalog number I/98A)

Macdonald, J., \& Mullan, D. J. 2010, ApJ, 723, 1599

Malo, L., Doyon, R., Feiden, G. A., et al. 2014, ApJ, 792, 37

Malo, L., Doyon, R., Lafrenière, D., et al. 2013, ApJ, 762, 88

Mann, A. W., Deacon, N. R., Gaidos, E., et al. 2014, AJ, 147, 160

Mann, A. W., Feiden, G. A., Gaidos, E., Boyajian, T., \& von Braun, K. 2015, ApJ, 804, 64

Mann, A. W., \& von Braun, K. 2015, PASP, 127, 102

Markwardt, C. B. 2009, in ASP Conf. Ser. 411, Astronomical Data Analysis Software and Systems, ed. D. A. Bohlender, D. Durand, \& P. Dowler (San Francisco, CA: ASP), 251

Mathieu, R. D., Baraffe, I., Simon, M., Stassun, K. G., \& White, R. 2007, Protostars and Planets V, ed. B. Reipurth, D. Jewitt, \& K. Keil (Tucson, AZ: Univ. Arizona Press), 411

McLean, M., Berger, E., Irwin, J., Forbrich, J., \& Reiners, A. 2011, ApJ, 741, 27

Melis, C., Reid, M. J., Mioduszewski, A. J., Stauffer, J. R., \& Bower, G. C. 2014, Sci, 345, 1029

Montagnier, G., Ségransan, D., Beuzit, J., et al. 2006, A\&A, 460, L19

Palla, F., Randich, S., Pavlenko, Y. V., Flaccomio, E., \& Pallavicini, R. 2007, ApJL, 659, L41

Preibisch, T., Brown, A. G. A., Bridges, T., Guenther, E., \& Zinnecker, H. 2002, AJ, 124, 404

Rajpurohit, A. S., Reylé, C., Allard, F., et al. 2013, A\&A, 556, A15

Rayner, J. T., Toomey, D. W., Onaka, P. M., et al. 2003, PASP, 115, 362

Rizzuto, A. C., Ireland, M. J., Dupuy, T. J., \& Kraus, A. L. 2016, ApJ, 817,164

Santos, N. C., Melo, C., James, D. J., et al. 2008, A\&A, 480, 889

Schaefer, G. H., Simon, M., Prato, L., \& Barman, T. 2008, AJ, 135, 1659

Schlieder, J. E., Bonnefoy, M., Herbst, T. M., et al. 2014, ApJ, 783, 27

Schlieder, J. E., Lépine, S., \& Simon, M. 2012, AJ, 143, 80

Service, M., Lu, J. R., Campbell, R., et al. 2016, PASP, 128, 095004

Simon, M., Dutrey, A., \& Guilloteau, S. 2000, ApJ, 545, 1034

Stassun, K. G., Feiden, G. A., \& Torres, G. 2014, NewAR, 60, 1

Stassun, K. G., Mathieu, R. D., \& Valenti, J. A. 2006, Natur, 440, 311

Stauffer, J. R., Schultz, G., \& Kirkpatrick, J. D. 1998, ApJL, 499, L199

Torres, C. A. O., Quast, G. R., Melo, C. H. F., \& Sterzik, M. F. 2008, in ASP Monograph Publications 5, Handbook of Star Forming Regions, Vol. II: The Southern Sky, ed. B. Reipurth (San Francisco, CA: ASP), 757

Vacca, W. D., Cushing, M. C., \& Rayner, J. T. 2003, PASP, 115, 389 van Leeuwen, F. 2009, A\&A, 497, 209

Williams, P. K. G., Berger, E., Irwin, J., Berta-Thompson, Z. K., \& Charbonneau, D. 2015, ApJ, 799, 192

Williams, P. K. G., Cook, B. A., \& Berger, E. 2014, ApJ, 785, 9

Wright, E. L., Eisenhardt, P. R. M., Mainzer, A. K., et al. 2010, AJ, 140, 1868 Yelda, S., Lu, J. R., Ghez, A. M., et al. 2010, ApJ, 725, 331

Zuckerman, B., \& Song, I. 2004, ARA\&A, 42, 685 